\title{
Comparative Assessment of the Harmonic Balance Navier Stokes Technology for Horizontal and Vertical Axis Wind turbine Aerodynamics
}

\author{
M. Sergio Campobasso ${ }^{\mathrm{a}, *}$, Jernej Drofelnik $^{\mathrm{b}}$, Fabio Gigante $^{\mathrm{b}}$ \\ ${ }^{a}$ University of Lancaster, Department of Engineering. Engineering Building, Gillow \\ Avenue, Lancaster LA1 $4 Y W$, United Kingdom. \\ ${ }^{b}$ University of Glasgow, School of Engineering. James Watt Building South, University \\ Avenue, Glasgow G12 8QQ, United Kingdom.
}

\begin{abstract}
Several important wind turbine unsteady flow regimes, such as those associated with the yawed wind condition of horizontal axis machines, and most operating conditions of all vertical axis machines, are predominantly periodic. The harmonic balance Reynolds-averaged Navier-Stokes technology for the rapid calculation of nonlinear periodic flow fields has been successfully used to greatly reduce runtimes of turbomachinery periodic flow analyses in the past fifteen years. This paper presents an objective comparative study of the performance and solution accuracy of this technology for aerodynamic analysis and design applications of horizontal and vertical axis wind turbines. The considered use cases are the periodic flow past the blade section of a utility-scale horizontal axis wind turbine rotor in yawed wind, and the periodic flow of a H-Darrieus rotor section working at a tip-speed ratio close to that of maximum power. The aforementioned comparative assessment is based on thorough parametric timedomain and harmonic balance analyses of both use cases. The paper also reports the main mathematical and numerical features of a new turbulent harmonic balance Navier-Stokes solver using Menter's shear stress transport model for the turbulence closure. Presented results indicate that a) typical multimegawatt
\end{abstract}

\footnotetext{
* Corresponding author

Email address: m.s.campobasso@lancaster.ac.uk (M. Sergio Campobasso)
} 
horizontal axis wind turbine periodic flows can be computed by the harmonic balance solver about ten times more rapidly than by the conventional timedomain analysis, achieving the same temporal accuracy of the latter method, and $b$ ) the harmonic balance acceleration for Darrieus rotor unsteady flow analysis is lower than for horizontal axis machines, and the harmonic balance solutions feature undesired oscillations caused by the wide harmonic content and the high-level of stall predisposition of this flow field type.

Keywords: Horizontal and vertical axis wind turbine periodic aerodynamics, Dynamic stall, Harmonic balance Navier-Stokes equations, Shear stress transport turbulence model, Fully coupled multigrid integration, Point-implicit Runge-Kutta smoother

\section{Nomenclature}

\section{Acronyms}

AoA Angle of attack

BEMT Blade element momentum theory

$5 \quad$ FERK Fully explicit Runge-Kutta

HAWT Horizontal axis wind turbine

HB Harmonic balance

IRS Implicit residual smoothing

MG Multigrid

10 NS Navier-Stokes

PDE Partial differential equation

PIRK Point-implicit RK

RK Runge-Kutta

TD Time-domain

15 VAWT Vertical axis wind turbine

\section{Greek symbols}

$\Delta \tau$ Pseudo-time-step $(s)$

$\Delta l_{r}$ Logarithm in base 10 of normalized residual RMS of RANS equations 
$\Omega$ Rotor angular speed $(R P M)$

${ }_{20} \quad \Omega_{f}$ Flow vorticity $\left(s^{-1}\right)$

$\underline{\Phi}_{c}$ Generalized steady and $T D$ convective flux vector

$\underline{\Phi}_{c H}$ Generalized $H B$ convective flux vector

$\underline{\Phi}_{d}$ Generalized steady and $T D$ diffusive flux vector

$\underline{\Phi}_{d H}$ Generalized $H B$ diffusive flux vector

${ }_{25} \alpha_{\infty}$ Angle of attack associated with velocity vector $\underline{W}_{\infty}\left({ }^{\circ}\right)$

$\alpha_{m} m^{\text {th }}$ RK coefficient

$\delta$ Yaw angle $\left(^{\circ}\right)$

$\gamma_{p}$ Twist angle $\left(^{\circ}\right)$

$\lambda$ Reduced frequency

30 $\quad \lambda_{D}$ Tip-speed ratio

$\mu_{T}$ Turbulent viscosity $(\mathrm{kg} / \mathrm{ms})$

$\nu$ Molecular kinematic viscosity $\left(\mathrm{m}^{2} / \mathrm{s}\right)$

$\omega$ Specific turbulence dissipation rate $\left(s^{-1}\right)$

$\phi_{\infty}^{r}$ Angle of attack associated with velocity vector $\underline{W}_{\infty}^{r}\left(^{\circ}\right)$

$35 \rho$ Density $\left(\mathrm{kg} / \mathrm{m}^{3}\right)$

$\tau_{w}$ Wall viscous stress $(\mathrm{Pa})$

$\theta$ VAWT rotor azimuthal position $\left(^{\circ}\right)$

\section{Latin symbols}

$A$ Matrix for implicit update of $k$ and $\omega$

${ }_{40} C D_{\omega}$ Cross-diffusion term of $\omega$ equation $\left(\mathrm{kg} / \mathrm{m}^{3} \mathrm{~s}^{2}\right)$

$C_{l}, C_{d}$ Lift and drag force coefficients

$C_{m}^{\prime}$ Constant-head pitching moment coefficient

$C_{m}$ Variable-head pitching moment coefficient

$C_{M G}$ Overhead of $H B \mathrm{MG}$ cycle

${ }_{45} \quad C_{T}$ Torque coefficient

$C_{x}, C_{y}$ Horizontal and vertical force coefficients

$D H B$ antisymmetric matrix

$D_{\omega}$ Destruction term of $\omega$ rate $\left(\mathrm{kg} / \mathrm{m}^{3} \mathrm{~s}^{2}\right)$

$D_{k}$ Destruction term of $k\left(\mathrm{~kg} / \mathrm{ms}^{3}\right)$ 
$M_{\infty}^{r}$ Mach number associated with velocity $\underline{W}_{\infty}^{r}$

$N_{H}$ Number of complex harmonics

$N_{\text {pde }}$ Number of PDEs

$P_{d}$ Turbulent production term $\left(s^{-2}\right)$

Q Array of steady and $T D$ conservative variables at cell center

${ }_{55} \mathbf{Q}_{H}$ Array of $H B$ conservative variables at cell center

$R$ HAWT rotor radius $(m)$

$\mathbf{R}_{\Phi}$ Array of steady and $T D$ cell residuals

$\mathbf{R}_{\Phi H}$ Array of $H B$ cell residuals

$R_{D}$ Darrieus turbine rotor radius $(\mathrm{mm})$

${ }_{60} \quad \mathbf{R}_{g H}$ Array of $H B$ cell residuals including $H B$ source term

S Source term of steady and $T D$ equations

$\mathbf{S}_{H}$ Source term of $H B$ equations

$S_{k}$ Source term of $k$ equation $\left(\mathrm{kg} / \mathrm{ms}^{3}\right)$

$S_{\omega}$ Source term of $\omega$ equation $\left(\mathrm{kg} / \mathrm{m}^{3} \mathrm{~s}^{2}\right)$

${ }_{65} T$ Period $(s)$

$\mathbf{U}$ Array of steady and $T D$ conservative variables

$\mathbf{U}_{H}$ Array of $H B$ conservative variables

$V_{\infty}$ Freestream velocity ahead of HAWT rotor $(\mathrm{m} / \mathrm{s})$

$\underline{W}_{\infty}$ Absolute freestream velocity vector $(\mathrm{m} / \mathrm{s})$

$70 \quad \underline{W}_{\infty}^{r}$ Relative freestream velocity vector $(\mathrm{m} / \mathrm{s})$

$c$ Chord $(m)$

$c_{f}, c_{p}$ Skin friction and static pressure coefficients

$k$ Turbulent kinetic energy $\left(\mathrm{m}^{2} / \mathrm{s}^{2}\right)$

$l$ RK cycle counter

${ }_{75} l_{k}$ User-given constant of turbulent production limiters

$m$ RK stage index

$p$ Static pressure $(\mathrm{Pa})$

$p_{w}$ Wall static pressure $(\mathrm{Pa})$

$\underline{\underline{s}}$ Strain rate tensor $\left(s^{-1}\right)$

so $t_{n} H B$ snapshot times 
v Local absolute velocity vector $(\mathrm{m} / \mathrm{s})$

$x_{a}$ Airfoil chorswise position $(m)$

$y^{+}$Dimensionless wall distance

\section{Introduction}

The aeromechanical design of wind turbines is a complex multidisciplinary task that requires consideration of a very large number of operating regimes due to the extreme variability of the environmental conditions on time scales ranging from seconds (e.g. wind gusts) to months (e.g. seasonal wind variations). Several fatigue-inducing unsteady regimes, however, are predominantly periodic. In the case of utility-scale horizontal axis wind turbines (HAWTs), periodic fluid-induced excitations of the rotor blades and drivetrain may result from the blades rotating $a$ ) through wind stratifications associated with the atmospheric boundary layer, $b$ ) through the variable pressure field due to the presence of the tower (multimegawatt turbines typically feature upwind rotors), c) through portions of the wake shed by an upstream turbine in the wind farm environment, $d$ ) in yawed wind, a condition occurring when the freestream wind velocity is not orthogonal to the turbine rotor [1], and $e$ ) in a region of nonuniform wind resulting from the combination of two or more of the kind of phenomena mentioned above. With regard to yaw misalignments, utility-scale HAWTs typically feature yaw control systems that monitor the direction of the wind and rotate the entire nacelle towards the wind [2]. However, yaw actuators adjust the nacelle position only after a yaw error has been detected for a relatively long time-interval, usually 10 minutes. Therefore, at sites with frequent variations of the wind direction, blade and drivetrain fatigue due to yawed wind can be significant. HAWT rotors experience constant periodic excitations when the turbines are placed at inclined sites, such as mountainous terrains. Here wind speeds are often higher than on flat terrain due to the acceleration induced by the surface geometry, however the entire wind stream is inclined on the ground, and this yields periodic rotor flows similar to those induced by 

excitation is a multiple of the rotor speed.

The flow field past vertical axis wind turbines (VAWTs) 2], such as the popular Darrieus turbine, is inherently unsteady and predominantly periodic in the vast majority of operating conditions. At present these machines are used predominantly for distributed power generation in the built environment. For this application, they are often preferred to HAWTs due their simpler build, simpler and cheaper maintenance requirements, and for their insensitivity to the wind direction. This feature is particularly important in the urban environment, as the variability of wind speed and direction is higher that on open terrains. The and rotate in planes orthogonal to the rotor axis. The periodic nature of the flow past the blades is due to the cyclic variation (every rotor revolution) of modulus and direction of the relative velocity perceived by their airfoils [4], and also the interactions between the blades traveling in the downwind region of the rotor and unsteady flow patterns are further complicated by the occurrence of dynamic stall [6] over a significant portion of the entire turbine operating range [5].

The comments above highlight the necessity of accurately predicting periodic flows when designing wind turbines. This is of crucial importance for reliably predicting the actual amount of harvested energy and the fatigue-inducing loads which may reduce turbine life and/or increase its operation and maintenance costs. In many cases, however, wind turbine design methods still rely on lowfidelity and/or semi-empirical models such as blade element momentum theory (BEMT) and dynamic stall models [7, 8, 9]. The main advantage of these techniques is their extremely high computational speed. Their main drawback is that they heavily rely on the existence and availability of high-quality airfoil data, and this hinders their applicability to the design of radically new turbine configurations. Moreover these low-fidelity methods model strongly unsteady three-dimensional (3D) flow features, such as HAWT yawed flows and the radial 
of uncertainty even when detailed airfoil data are available. A wider discussion on the predictive reliability of low-fidelity tools for the wind turbine design can be found in [11].

The use of high-fidelity computational aerodynamics tools such as NavierStokes (NS) Computational Fluid Dynamics (CFD) codes has the potential of greatly reducing the uncertainty associated with the flow predictions of lowfidelity models. Several remarkable examples of the predictive capabilities of NS CFD for HAWT yawed flows have been published, including the articles [12, 13, 14, 15]. The article [12] also includes comparisons of CFD NS results, experimental data and results obtained with low-fidelity codes, including a BEMT code. The report shows that the agreement between NS CFD analysis and measured data is substantially better than that between low-fidelity analyses and measured data, as expected. Early assessments of the NS CFD technology for Darrieus rotor aerodynamics, aiming primarily at thoroughly investigating the complex fluid mechanics of these machines, include the articles [16, 5, 6]. The computational and experimental study reported in [17] provides detailed evidence of the predictive capabilities of 3D NS CFD for Darrieus rotors. An exhaustive comparative assessment of NS CFD and BEMT results, highlighting the difficulties of the BEMT technology of accurately predicting complex flow features, particularly in the absence of reliable airfoil force data, is reported in [18]. The article 19] also highlights that NS codes can predict fairly accurately measured Darrieus turbine aerodynamics provided that best practice in defining the physical domain, constructing the computational grid, and setting up important parameters of the simulation is adopted.

The main drawback of NS simulations is their high computational cost. A fully time-resolved time-domain $(T D)$ NS simulation of wind turbine periodic flows requires a long runtime because several rotor revolutions have to be simulated before the periodic state of interest is achieved. This runtime could be reduced by using a frequency-domain formulation and solution of the governing unsteady equations. The harmonic balance $(H B)$ NS technology for the solution of unsteady periodic flows [20] is one of the most popular technologies 
of this type. This method has been successfully applied to the prediction of the periodic flow associated with flutter and forced response of turbomachinery blades 20, 21, 22], and various vibratory motion modes of aircraft configurations [23, 24, 25]. For this type of application, the use of the $H B$ NS approach for the calculation of periodic flows can lead to runtime reductions varying between one and two orders of magnitude with respect to conventional TD NS analyses. Other successful nonlinear frequency-domain NS methods exist and have been used, and more detail on this aspect can be found in [26].

A preliminary investigation into the use of the $H B$ NS technology for reducing the analysis runtime of the periodic flow field past HAWT rotor blade sections was reported in [26]. This study was based on the compressible laminar NS equations and used low-speed preconditioning to handle the numerical difficulties resulting from the typically low speeds of wind turbine flows. More realistic turbulent flow demonstrations of this technology for HAWT turbulent aerodynamics have followed, including the study in 27] making use of the one-equation Spalart-Allmaras turbulence model [28], that in [29] making use of the Spalart-Allmaras model and a zonal transition model, and that in [11] making use of Menter's two-equation shear stress transport (SST) turbulence model [30]. The only reported study on the use of the NS $H B$ technology for VAWT aerodynamics is, to the best of the authors' knowledge, the article [31], which presents parametric design studies of a one-blade Darrieus rotor based on a $H B$ NS code making use of an algebraic model for the turbulence closure. These studies indicate a growth in the use of this high-fidelity approach for the analysis of HAWT periodic aerodynamics. However, quantitative measures of the actual benefits of using turbulent $H B$ NS solvers for wind turbine design are still scarce. More specifically, by which amount can a turbulent $H B$ NS code reduce the analysis runtime of wind turbine periodic flows while maintaining a prediction accuracy comparable to that of the corresponding TD code? Can both HAWT and Darrieus VAWT flows be solved with an accuracy comparable to that of the $T D$ method, but more rapidly? The main objective of this paper is to provide a significant contribution to answering these questions. 
After presenting the $T D$ and $H B$ integral form of the Reynolds-averaged Navier-Stokes (RANS) equations and the SST turbulence model used for the turbulence closure (section 2), brief descriptions of the multigrid smoother of the steady and $H B$ solvers of the COSA NS research code are provided (section 3). Here emphasis is put on the strongly coupled integration approach of all COSA solvers, which advance concurrently in the integration process the solution of the two systems of algebraic equations resulting from the discretization of the RANS and SST equations. Section 4 considers the periodic flow past the blade section of a utility-scale HAWT. In addition to providing a detailed aerodynamic discussion of this flow problem, time refinement analyses with the TD solver and spectral refinement analyses with the $H B$ solver are performed to determine the speed-up of the $H B$ simulation yielding a solution accuracy comparable 215 to that of the fully resolved $T D$ simulation. The same type of analyses for the periodic flow of a three-blade H-Darrieus rotor section are presented in section 5. The paper is concluded by a summary of the presented analyses and a some perspectives on the future use of the $H B$ NS technology for wind turbine aerodynamics.

\section{Governing equations}

\subsection{Time-domain formulation}

The compressible NS equations are a system of nonlinear partial differential equations (PDEs) expressing the conservation of mass, momentum and energy in a viscous fluid flow. Averaging the NS equations on the longest time-scales of turbulence yields the so-called RANS equations, which feature additional terms depending on the Reynolds stress tensor. Making use of Boussinesq approximation, this tensor is expressed as the product of the strain rate tensor and a turbulent or eddy viscosity. In the COSA CFD code, the latter variable is computed by means of the two-equation $k-\omega$ SST turbulence model. Thus, turbulent flows are determined by solving a system of $N_{p d e}=6$ PDEs in two dimensions and $N_{p d e}=7$ in three dimensions. 
Given a moving control volume $\mathcal{C}$ with time-dependent boundary $S(t)$, the Arbitrary Lagrangian-Eulerian integral form of the system of the time-dependent RANS and SST equations is:

$$
\frac{\partial}{\partial t}\left(\int_{\mathcal{C}(t)} \mathbf{U} d \mathcal{C}\right)+\oint_{S(t)}\left(\underline{\boldsymbol{\Phi}}_{c}-\underline{\mathbf{\Phi}}_{d}\right) \cdot d \underline{S}-\int_{\mathcal{C}(t)} \mathbf{S} d \mathcal{C}=0
$$

235 where $\mathbf{U}=\left[\begin{array}{lllll}\rho & \rho \underline{\mathrm{v}}^{\prime} & \rho E & \rho k & \rho \omega\end{array}\right]^{\prime}$ is the array of conservative variables, $\rho$, $\underline{\mathrm{v}}, E, k$ and $\omega$ are, respectively, the flow density, the flow velocity vector, the total energy per unit mass, the turbulent kinetic energy per unit mass and the specific dissipation rate of turbulent energy, and the superscript ' denotes the transpose operator. The total energy is $E=e+(\underline{\mathrm{v}} \cdot \underline{\mathrm{v}}) / 2+k$, where $e$ denotes the internal energy per unit mass; the perfect gas law is used to express the static pressure $p$ as a function of $\rho, E, k$ and the mean flow kinetic energy per unit mass $(\underline{\mathrm{v}} \cdot \underline{\mathrm{v}}) / 2$. The expressions of the generalized convective flux vector $\underline{\boldsymbol{\Phi}}_{c}$ and the generalized diffusive flux vector $\underline{\Phi}_{d}$ are reported in [11].

The turbulent viscosity $\mu_{T}$, required to calculate the Reynolds stress tensor [11], is given by

$$
\mu_{T}=a_{1} \rho k / \max \left(a_{1} \omega, F_{2}\left|\Omega_{f}\right|\right)
$$

in which $a_{1}=0.31, \Omega_{f}$ is the flow vorticity, and $F_{2}$ is a function of $k, \omega$, the molecular kinematic viscosity $\nu$ and the distance from the wall $d$. The expression of $F_{2}$ can be found in [30].

The definition of the source term $\mathbf{S}$ in Eqn. (10) is $\mathbf{S}=\left[\begin{array}{lllll}0 & \underline{0^{\prime}} & 0 & S_{k} & S_{\omega}\end{array}\right]^{\prime}$ where

$$
\begin{aligned}
S_{k} & =\mu_{T} P_{d}-\frac{2}{3}(\nabla \cdot \underline{\mathrm{v}}) \rho k-D_{k} \\
S_{\omega} & =\gamma \rho P_{d}-\frac{2}{3}(\nabla \cdot \underline{\mathrm{v}}) \frac{\gamma \rho k}{\nu_{T}}-D_{\omega}+C D_{\omega}
\end{aligned}
$$

and

$$
\begin{gathered}
P_{d}=2\left[\underline{\underline{s}}-\frac{1}{3} \nabla \cdot \underline{\mathrm{v}}\right] \nabla_{\underline{\mathrm{v}}} \\
D_{k}=\beta^{*} \rho k \omega \quad D_{\omega}=\beta \rho \omega^{2} \\
C D_{\omega}=2\left(1-F_{1}\right) \rho \sigma_{\omega 2} \frac{1}{\omega} \nabla k \cdot \nabla \omega
\end{gathered}
$$


Here $\nu_{T}$ is the turbulent kinematic viscosity, and the variables $\sigma_{k}, \sigma_{\omega}, \gamma, \beta^{*}$ and $\beta$ are weighted averages of the constants of the standard $k-\omega$ model [32] and the constants of the standard $k-\epsilon$ model [33] with weights $F_{1}$ and $\left(1-F_{1}\right)$, respectively. The function $F_{1}$ depends on the local values of $k, \omega, \nu, \rho, d, \nabla k$ and $\nabla \omega[30], \sigma_{\omega 2}$ is a constant of the standard $k-\epsilon$ model, and the symbol $\underline{\underline{s}}$ denotes the strain rate tensor, defined as $\underline{\underline{s}}=\left(\nabla \underline{\mathrm{v}}+\nabla \underline{\mathbf{v}}^{\prime}\right) / 2$.

It can be shown that the production term $P_{d}$ is always positive. Thus the source term $S_{k}$ of the $k$-equation has a term which is always positive (production term proportional to $P_{d}$ ), a term which is always negative (destruction term $D_{k}$ ) and a term which is positive or negative depending on the sign of $\nabla \cdot \underline{v}$. Similarly to $S_{k}$, the source term $S_{\omega}$ of the $\omega$-equation also has a term which is always positive (production term proportional to $P_{d}$ ), a term which is always negative (destruction term $D_{\omega}$ ), and a term which is positive or negative depending on the sign of $\nabla \cdot \underline{\mathrm{v}}$. The source term $S_{\omega}$, however, features an additional cross-diffusion term $C D_{\omega}$ which can be positive or negative. As seen below, the identification of positive and negative source terms is of crucial importance when using a point-implicit integration of the equations of turbulence [34, 35],

\subsection{Harmonic balance formulation}

The derivation of the high-dimensional $H B$ formulation [36] of the RANS and SST equations follows the same steps of that of the high-dimensional $H B$ NS equations [26], and yields:

$$
\Omega D\left(\int_{\mathcal{C}_{H}} \mathbf{U}_{H} d \mathcal{C}_{H}\right)+\oint_{S_{H}}\left(\underline{\mathbf{\Phi}}_{c H}-\underline{\mathbf{\Phi}}_{d H}\right) \cdot d \underline{S}_{H}-\int_{\mathcal{C}_{H}} \mathbf{S}_{H} d \mathcal{C}_{H}=0
$$

where $\Omega$ is the known excitation frequency, $D$ is the $\left(N_{\text {eqs }} \times N_{\text {eqs }}\right)$ antisymmetric matrix with $N_{\text {eqs }}=\left[N_{\text {pde }} \times\left(2 N_{H}+1\right)\right]$ defined in [26], and $N_{H}$ is the usergiven number of complex harmonics retained in the truncated Fourier series approximating the sought periodic flow field, The unknown array $\mathbf{U}_{H}$ is made up of $2 N_{H}+1$ flow states or snapshots, referring to the equally spaced points of one period:

$$
t_{n}=\frac{n}{\left(2 N_{H}+1\right)} \frac{2 \pi}{\Omega}, \quad n=0,1, \ldots, 2 N_{H}
$$


and its definition is therefore $\mathbf{U}_{H}=\left[\mathbf{U}\left(t_{0}\right)^{\prime} \mathbf{U}\left(t_{1}\right)^{\prime} \ldots \mathbf{U}\left(t_{N_{H}}\right)^{\prime}\right]^{\prime}$. The structure

of $\underline{\boldsymbol{\Phi}}_{c H}, \underline{\boldsymbol{\Phi}}_{d H}, \mathbf{S}_{H}, \mathcal{C}_{H}$ and $d \underline{S}_{H}$ is similar to that of $\mathbf{U}_{H}$.

The high-dimensional $H B$ method represents the frequency-domain governing equations in the time-domain, where they take the form of a set of coupled steady problems. Passing from the conventional time-domain framework to the harmonic balance framework, the number of PDEs increases from $N_{p d e}$ to $\left[N_{p d e} \times\left(2 N_{H}+1\right)\right]$. Despite this, however, the $H B$ approach allows turbulent periodic flows to be computed at a significantly lower computational cost than with the $T D$ approach in many problems of engineering interest.

\section{Numerical method}

\subsection{Space discretization}

The finite volume cell-centered parallel CFD code COSA [37, 26, 38, 39] solves the integral form of both the $T D$ conservation laws (System (11)) and the $H B$ conservation laws (System (8) ) using structured multi-block grids. In moving-body problems, the governing equations are solved in the absolute frame of reference, where the whole computational grid moves with a rigid body motion conforming to the user-given motion of the considered geometry (e.g. rotor blade).

The discretization of the convective fluxes of both RANS and SST PDEs is based on Van Leer's second order MUSCL extrapolations and Roe's fluxdifference splitting. Van Albada's flux limiter has been used for all simulations reported in this paper. The discretization of the diffusive fluxes and the turbulent source terms is based on second order finite-differencing, as reported in [37]. That article also provides the definitions of the viscous wall and far field boundary conditions used by COSA.

For steady problems the time-derivative appearing in System (1) vanishes and, for each cell of a computational grid, the discretized form of that system of PDEs becomes a system of $N_{p d e}$ nonlinear algebraic equations of the form:

$$
\mathbf{R}_{\Phi}(\mathbf{Q})=0
$$


The $N_{\text {pde }}$ entries of $\mathbf{Q}$ are the unknown conservative variables at the cell center, whereas the $N_{p d e}$ entries of $\mathbf{R}_{\Phi}$ store the cell residuals.

\subsection{Integration of steady equations}

The RANS and SST equations are solved with a pseudo-time-marching algorithm using the so-called fully coupled approach [34, 35] whereby the two sets of equations are time-marched simultaneously. The unknown flow vector $\mathbf{Q}$ is computed by solving iteratively Eqn. (10). A fictitious time-derivative $(d \mathbf{Q} / d \tau)$ premultiplied by the cell volumes is added to this system, and this derivative is then discretized with a four-stage Runge-Kutta (RK) scheme. The numerical solution is time-marched until the steady state is achieved. The convergence rate is enhanced by means of local time-stepping, variable-coefficient central implicit residual smoothing (IRS) and a full-approximation scheme multigrid (MG) algorithm. When solving turbulent problems using a two-equation turbulence model, however, this integration method becomes numerically inefficient due to the operator stiffness associated with the large negative source terms of the turbulence model. To alleviate this problem, a point-implicit integration strategy is adopted [34], whereby the abovesaid source terms are treated implicitly within each RK stage. Adopting this approach (see [37] for the detailed derivation), the steady turbulent point-implicit RK (PIRK) smoother reads:

$$
\begin{aligned}
& \mathbf{W}^{0}=\mathbf{Q}_{l} \\
& \left(I+\alpha_{m} \Delta \tau A\right) \mathbf{W}^{m}=\mathbf{W}^{0}+\alpha_{m} \Delta \tau A \mathbf{W}^{m-1}- \\
& \quad \alpha_{m} \Delta \tau V^{-1} L_{I R S}\left[\mathbf{R}_{\Phi}\left(\mathbf{W}^{m-1}\right)+\mathbf{f}_{M G}\right], \quad m=1,4 \\
& \mathbf{Q}_{l+1}=\mathbf{W}^{M}
\end{aligned}
$$

where $\Delta \tau$ is the local pseudo-time-step, $V$ is the cell volume, $l$ is the RK cycle counter, $m$ is the RK stage index, $\alpha_{m}$ is the $m^{\text {th }} \mathrm{RK}$ coefficient, $L_{I R S}$ denotes the IRS operator, and $\mathbf{f}_{M G}$ is the MG forcing function. The only nonzero elements of the $\left(N_{p d e} \times N_{p d e}\right)$-matrix $A$ are the elements of an upper triangular matrix making up its bottom right $(2 \times 2)$ partition, given by:

$$
A(5: 6,5: 6)=\left[\begin{array}{cc}
\left(\Delta^{+}+\beta^{*} \omega\right) & \beta^{*} k \\
0 & \gamma \Delta^{+}+2 \beta \omega
\end{array}\right]
$$


in which $\Delta^{+}=\max \left(0, \frac{2}{3} \nabla \cdot \underline{\mathrm{v}}\right)$. Eqn. (12) is the exact term resulting from the point-implicit integration of the $k-\omega$ model, but it is instead an approximation in the case of the SST model. The exact term for the SST case has also a nonzero $(5,6)$ entry [37]. Numerical experiments, however, reveal that the results computed with either Eqn. (12) or the exact matrix partition $A(5: 6,5: 6)$ of the SST model differ by negligible amounts. The use of Eqn. (12) also enables to update $\rho k$ and $\rho \omega$ using successive substitutions and avoiding more costly matrix inversions. For these reasons, COSA uses Eqn. (12) also for the SST model.

In order to prevent the specific dissipation rate $\rho \omega$ from taking unphysically low values, the value of this variable obtained with Algorithm [1] is limited by the minimum threshold:

$$
(\rho \omega)_{\min }=\gamma \rho \sqrt{P_{d}}
$$

as suggested in [34]. In the authors' experience, the use of Eqn. (13) yields substantial improvements of the numerical stability of the presented integration approach for most turbulent problems considered thus far, including those reported in this article. It is also noted that the partial decoupling of the update process of the two turbulent variables enabled by the upper triangular form of $A(5: 6,5: 6)$ allows a straightforward application of the constraint expressed by Eqn. (13): one first updates $\rho \omega$ with the sixth component of one PIRK stage, 350 then constrains the new value of $\rho \omega$ with Eqn. (13), and finally updates $\rho k$ with the fifth component of the same PIRK stage making use of the constrained $\rho \omega$. Thus far, the authors' experiments aimed at incorporating Eqn. (13) in the exact formulation of the SST variables update, which features a nonzero $A(6,5)$, have resulted in a dramatic reduction of the numerical stability of the fully coupled integration of the RANS and SST equations. Such stability reduction is even stronger for time-domain and harmonic balance problems, and for this reason COSA uses Eqn. (12) also for these simulations.

In the present implementation of the SST turbulence model, the production terms of both $k$ and $\omega$ are limited with an approach similar to that proposed 

production term $P_{d}$ appearing in Eqns. (3) and (4):

$$
\tilde{P}_{d}=\min \left(P_{d}, \frac{l_{k} D_{k}}{\mu_{t}}\right)
$$

where $\tilde{P}_{d}$ is the limited production term used to build the residuals of $k$ and $\omega$, and $l_{k}$ is a user-given constant. The article [40] proposes $l_{k}=20$, and reports that this limiter has two functions, namely to: a) 'eliminate the occurrence tensor $\underline{\underline{s}}$, and b) 'eliminate the unphysical build-up of eddy viscosity in the stagnation region of an airfoil'. For relatively simple problems, the solutions obtained with and without the use of Eqn. (14) differ very little. However, in the case of complex unsteady flows, such as those associated with VAWT rotors, the solutions obtained with and without the limiter of Eqn. (14) may differ significantly, as observed in section 5 .

The integration of the TD RANS and SST equations is accomplished by using the same strongly coupled point-implicit approach reported above within a dual-time-stepping algorithm with second order accuracy in the physical time. discretization of the physical time-derivative of both RANS and SST equations is also adopted [41] (the algorithmic details are provided in [37]. The resulting $T D$ PIRK smoother enables the use of higher Courant Friedrichs Lewis (CFL) numbers than the conventional TD fully explicit RK (FERK) smoother [37, 38]. The TD PIRK approach does not require any additional costly operation with respect to the TD FERK approach. Thus, the TD PIRK method reduces the simulation runtime proportionally to the reduction of the the MG cycles required to achieve a prescribed reduction of the residuals.

Since the PIRK treatment of the negative source terms of the SST model is used by all COSA solvers, in the remainder of this report the acronyms PIRK and FERK will be used only with reference to the numerical treatment of the unknown source term arising from the discretization of the time-derivative in the $T D$ equations, and the source term associated with the $H B$ counterpart 
of the time-derivative of the $T D$ equations (this term is introduced in next subsection).

\subsection{Integration of harmonic balance equations}

The only difference between Systems (10) and (8) is that the physical timederivative of the former system is replaced by a volumetric source term proportional to $\Omega$ in the latter. The set of nonlinear algebraic equations resulting from the space discretization of System (8) is thus solved with the same technique used for steady problems. The $H B$ solution $\mathbf{Q}_{H}$ at each cell center is obtained by solving the system of algebraic equations:

$$
\mathbf{R}_{g H}\left(\mathbf{Q}_{H}\right)=\Omega V_{H} D \mathbf{Q}_{H}+\mathbf{R}_{\Phi H}\left(\mathbf{Q}_{H}\right)=0
$$

The array $\mathbf{Q}_{H}$ is made up of $\left(2 N_{H}+1\right)$ flow states, each referring to the physical times defined by Eqn. (9), and has length $\left[N_{p d e} \times\left(2 N_{H}+1\right)\right]$. The first $N_{p d e}$ elements of $\mathbf{Q}_{H}$ contain the flow state at $t=t_{0}$, the next $N_{p d e}$ elements contain the flow state at $t=t_{1}$, and so on. The arrays $\mathbf{R}_{g H}$ and $\mathbf{R}_{\Phi H}$ have the same structure of $\mathbf{Q}_{H}$. The $\left(2 N_{H}+1\right)$ states of $\mathbf{R}_{\Phi}$ contain the residuals associated with the convective fluxes, the diffusive fluxes and the turbulent source terms at the considered physical times. The residual array $\mathbf{R}_{g}$ also includes the source term $\Omega V_{H} D \mathbf{Q}_{H}$, where $V_{H}$ is an array containing the values of the cell volume at the considered times.

The $H B$-counterpart of the turbulent steady smoother (11) is:

$$
\begin{aligned}
& \mathbf{W}_{H}^{0}=\left(\mathbf{Q}_{H}\right)_{l} \\
& {\left[I+\alpha_{m} \Delta \tau_{H} A_{H}\right] \mathbf{W}_{H}^{m}=\mathbf{W}_{H}^{0}+\alpha_{m} \Delta \tau_{H} A_{H} \mathbf{W}_{H}^{m-1}-} \\
& \quad \alpha_{m} \Delta \tau_{H} V_{H}^{-1} L_{I R S, H}\left[\mathbf{R}_{g_{H}}\left(\mathbf{W}_{H}^{m-1}\right)+\mathbf{f}_{M G, H}\right], \quad m=1,4 \\
& \left(\mathbf{Q}_{H}\right)_{l+1}=\mathbf{W}_{H}^{M}
\end{aligned}
$$

where the array $\Delta \tau_{H}$ has $\left(2 N_{H}+1\right)$ entries containing the local time-steps for the $2 N_{H}+1$ flow states. The $H B \mathrm{MG}$ forcing term $\mathbf{f}_{M G, H}$ has the same structure of $\mathbf{Q}_{H}$. The block-diagonal matrix $A_{H}$ has $2 N_{H}+1$ blocks of dimension [ $\left.N_{\text {pde }} \times N_{\text {pde }}\right]$, each referring to one of the $2 N_{H}+1$ states. The structure of 
each block is the same as that of the matrix $A$ in Algorithm (11). The $H B$ IRS operator $L_{I R S, H}$ has the same block structure of $A_{H}$.

When using Eqn. (12) for the update of $\rho k$ and $\rho \omega$, the structure of the matrix premultiplying $\mathbf{W}_{H}^{m}$ at the second line of Algorithm (16) is such that, for each grid cell, the update of the $\left[N_{p d e} \times\left(2 N_{H}+1\right)\right]$ unknowns does not require any matrix inversion. It is also noted that Algorithm (16) uses a FERK treatment of the $H B$ source term $\Omega V_{H} D \mathbf{Q}_{H}$. In the light of the superior convergence rate of the PIRK over the FERK integration for turbulent $T D$ flows solved with the RANS and SST equations [37], it is expected that a point-implicit treatment of the $H B$ source term [11] may enable the use of larger CFL numbers, thus further increasing the convergence rate of the $H B$ equations. The $H B$ PIRK integration, however, increases the computational cost of each RK stage, because, for each cell, it requires the inversion of two matrices of size [ $\left(2 N_{H}+\right.$ $\left.\left.{ }_{425} 1\right) \times\left(2 N_{H}+1\right)\right]$ for updating $k$ and $\omega$. The convenience of the approach depends on whether the faster convergence enabled by higher CFL numbers outweighs the additional burden of the matrix inversions. This feature is case-dependent, and for all simulations reported in this article, the $H B$ PIRK integration did not enable the use of CFL numbers higher than those used by the $H B$ FERK approach of Algorithm (16).

It is also noted that the ratio of the computational cost of one $H B$ FERK MG cycle and that of one steady MG cycle grows in a slightly superlinear fashion with $N_{H}$, due to construction of the $H B$ source term $V_{H} D_{H} \mathbf{Q}_{H}$. This overhead, however, remains relatively small even for very high values of $N_{H}$ up to 16 , as highlighted in the numerical tests provided below.

\section{Horizontal axis wind turbine blade section}

All COSA solvers have been thoroughly verified and validated as reported in [11, 37, 26, 38, 42]. This section presents the analysis of the two-dimensional (2D) turbulent periodic flow past the airfoil of a rotating HAWT blade in yawed wind. The rotor radius is $82.0 \mathrm{~m}$ and the rotor speed is $12.0 R P M$, which 
corresponds to a value of $\Omega$ of about $1.26 \mathrm{rad} / \mathrm{s}$. The freestream wind velocity $V_{\infty}$ is $13 \mathrm{~m} / \mathrm{s}$, and a yaw angle $\delta$ of $45^{\circ}$ is assumed. The considered section is at a distance $R$ of $24.6 \mathrm{~m}$ (30\% rotor radius) from the rotational axis, and it has a chord $c$ of $5.2 \mathrm{~m}$ and a twist $\gamma_{p}$ of $10.44^{\circ}$. The 2D analysis set-up is obtained using the yawed wind reduction model reported in [11], to which the reader is referred for further detail. Making use of that model, the yawed wind condition perceived by the airfoil at rotor radius $R$ can be approximated by the unsteady 2D flow field resulting from a horizontal harmonic motion of the airfoil in a steady freestream at speed $W_{\infty}$ and direction $\alpha_{\infty}$, respectively given by:

$$
\begin{aligned}
W_{\infty} & =\sqrt{\left(V_{\infty} \cos \delta\right)^{2}+(\Omega R)^{2}} \\
\alpha_{\infty} & =\arctan \left[\left(V_{\infty} \cos \delta\right) /(\Omega R)\right]
\end{aligned}
$$

Using these equations, one finds $W_{\infty}=32.2 \mathrm{~m} / \mathrm{s}$ and $\alpha_{\infty}=16.56^{\circ}$. Choosing the standard temperature of $288 \mathrm{~K}$, the Mach number $M_{\infty}$ corresponding to $W_{\infty}$ and adopted in the 2D simulations is 0.095 . In the $2 \mathrm{D}$ model, the mesh is built past the twisted airfoil, and the angle $\phi_{\infty}$ between the freestream at speed $W_{\infty}$ and the chord (angle of attack) is thus $\phi_{\infty}=\alpha_{\infty}-\gamma_{p}=6.12^{\circ}$. The expression of the harmonic motion is:

$$
\begin{aligned}
h(t) & =h_{0} \sin (\Omega t) \\
h_{0} & =V_{\infty} \sin \delta / \Omega
\end{aligned}
$$

Each period of the 2D harmonic motion corresponds to a revolution of the turbine rotor. Inserting the data provided above into Eqn. (20) gives $h_{0}=1.4 \mathrm{c}$. The reduced frequency is $\lambda=\Omega c / W_{\infty}=0.203$.

The blade section features the DU99 W 350LM airfoil, which has a maximum thickness-to-chord ratio of 35 percent. The Reynolds number based on the standard density of $1.22 \mathrm{~kg} / \mathrm{m}^{3}$, the velocity $W_{\infty}$, the airfoil chord and the air viscosity at standard temperature is $1.15 \times 10^{7}$. The 524,288 -cell C-grid adopted for all simulations has 512 mesh intervals along the airfoil, 256 intervals in the grid cut, and 512 intervals in the normal-like direction. The far field boundary ${ }_{465}$ is at about 50 chords from the airfoil, and the distance $d_{w}$ of the first grid points 


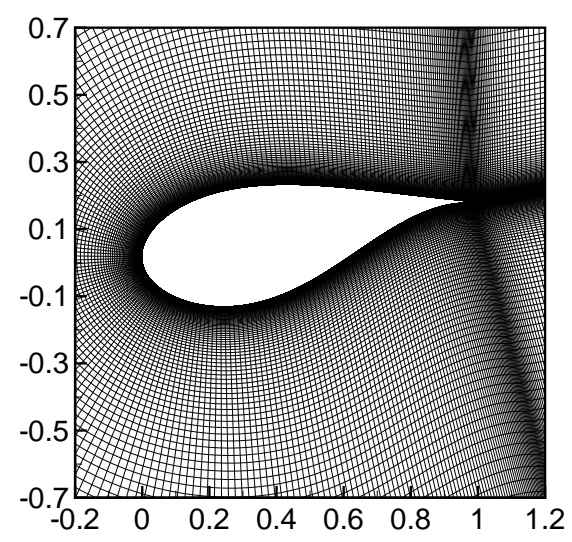

Figure 1: HAWT blade section test case: grid view in airfoil region (only every second line in both directions is plotted).

off the airfoil surface from the surface itself is about $10^{-6} c$. The nondimensional minimum distance from the wall is $y^{+}=\left(u_{\tau} d_{w}\right) / \nu_{w}$, where $u_{\tau}$ is the friction velocity and $\nu_{w}$ is the kinematic viscosity at the wall. In all the simulations reported below, the maximum value of $y^{+}$was always smaller than 1 .

As mentioned above, the airfoil and the whole grid are inclined by the twist angle $\gamma_{p}$ on the horizontal direction, and Fig. 1 provides an enlarged view of the adopted grid in the airfoil region. For visual clarity, only every second line of both grid line sets is plotted. In the unsteady simulations, the whole grid undergoes a sinusoidal motion defined by Eqn. (19), with amplitude $h_{0}$ given by Eqn. (20). All steady, $T D$ and $H B$ simulations have been performed using the MG solver with 3 grid levels. No CFL ramping has been used, and the CFL number has been set to 4 from the beginning of all simulations.

\subsection{Aerodynamic analyses}

To determine the minimal time-resolution of the $T D$ analysis required to obtain a solution independent of further reductions of the physical time-step, four different $T D$ simulations have been performed using a number of physical time-steps per period $N_{p}$ of $256,128,64$, and 32 . In the discussion below, 
these simulations are denoted by $T D N_{p}$. Three force coefficients have been monitored in the simulations: the horizontal force coefficient $C_{x}$, the vertical force coefficient $C_{y}$, and the constant-head pitching moment coefficient $C_{m}^{\prime}$, defined respectively as:

$$
C_{x}=\frac{F_{x}}{0.5 \rho_{\infty} W_{\infty}^{2} c} \quad C_{y}=\frac{F_{y}}{0.5 \rho_{\infty} W_{\infty}^{2} c} \quad C_{m}^{\prime}=\frac{M}{0.5 \rho_{\infty} W_{\infty}^{2} c^{2}}
$$

The horizontal force per unit blade length $F_{x}$ is the tangential force component that results in useful torque; the vertical force per unit blade length $F_{y}$ is the axial force component that results in rotor thrust; the pitching moment per unit blade length $M$ at one quarter chord from the leading edge provides a measure of the torsional aerodynamic load on the blade. All four $T D$ simulations have been run until the maximum $C_{x}, C_{y}$ and $C_{m}^{\prime}$ differences over two consecutive oscillation cycles became less than $0.1 \%$ of their maxima over the latter cycle of the cycle pair.

The coefficients $C_{x}, C_{y}$ and $C_{m}^{\prime}$ are all constant-head force coefficients. A variable-head force coefficient set is also considered below, namely the standard lift force coefficient $C_{l}$, the drag coefficient $C_{d}$, and the quarter-chord pitching moment coefficient $C_{m}$. The force coefficients $C_{l}$ and $C_{d}$ differ from $C_{x}$ and $C_{y}$ not only because they consider different force components, but also because the dynamic head at the denominator of $C_{l}$ and $C_{d}$ is that associated with the relative time-dependent freestream velocity $\underline{W}_{\infty}^{r}$, which has components

$$
W_{x}=\Omega R-V_{\infty} \sin (\delta) \cos (\Omega t), \quad W_{y}=V_{\infty} \cos (\delta)
$$

and forms an angle $\alpha_{\infty}^{r}$ with the horizontal direction given by:

$$
\alpha_{\infty}^{r}=\arctan \left(W_{y} / W_{x}\right)
$$

The coefficients $C_{m}$ and $C_{m}^{\prime}$ also differ because of the different definition of the dynamic head. It should also be noted that the directions of lift and drag change throughout the period, due to the time-dependence of $\alpha_{\infty}^{r}$, whereas the directions of $F_{x}$ and $F_{y}$ are constant. More detail on this aspect can be found in 11]. 
The $C_{l}, C_{d}$ and $C_{m}$ profiles over one revolution computed by the four $T D$ analyses are depicted in the three subplots of Fig. 2. The variable along the

rear portion of the airfoil pressure side at 30 percent of the period is higher than at 70 percent of the period. It should be noted that, since the dynamic head and the relative flow direction used to compute the $C_{l}, C_{d}$ and $C_{m}$ coefficients 
vary during the period, these coefficients do not provide a direct measure of the section contribution to the aerodynamic loads acting on the blade. Direct measures of the forces acting on the blade section are instead provided by the constant-head coefficients $C_{x}, C_{y}$ and $C_{m}^{\prime}$ examined later in this subsection.
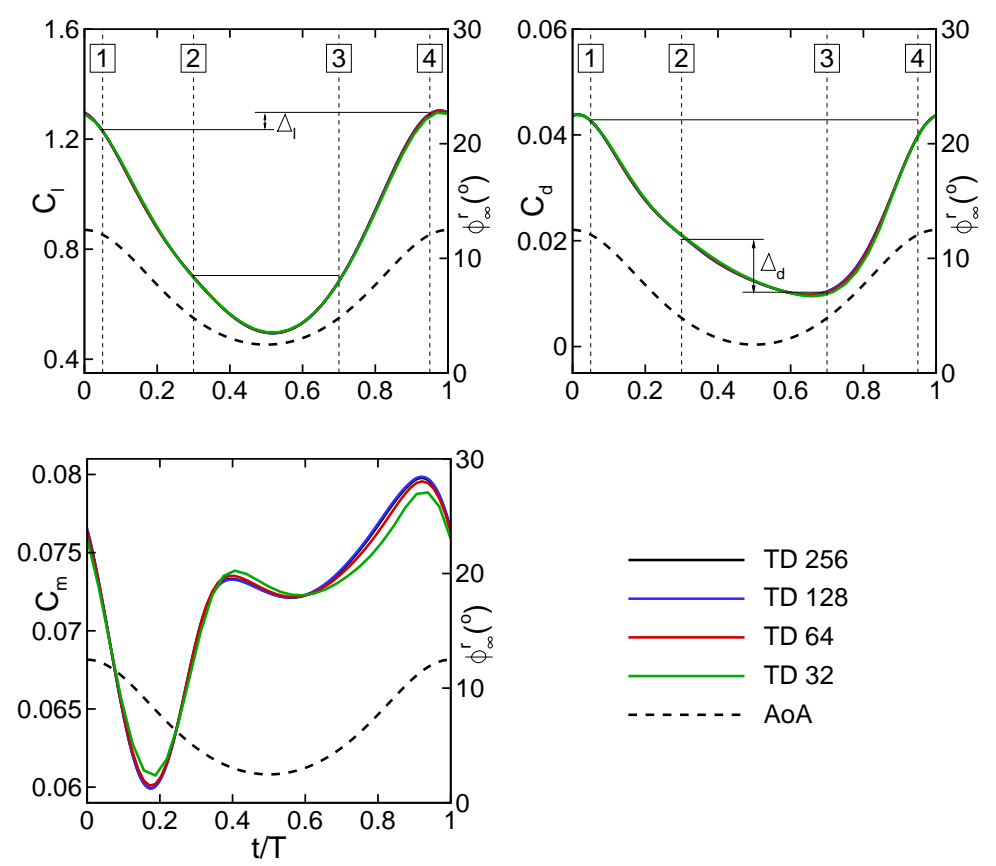

Figure 2: HAWT blade section test case: periodic profiles of variable head force coefficients over one period computed with four $T D$ analyses. Top left: lift coefficient; top right: drag coefficient; bottom left: pitching moment coefficient.

The four subplots of Fig. 3 show the TD 128 contours of flow vorticity $\Omega_{f}$ and the streamlines past the blade section at the positions labeled 1 to 4 in Fig. 2 The top left and bottom right subplots refer respectively to the positions at 5 and 95 percent of the period, and their comparison confirms that the amount of flow reversal in the rear portion of the airfoil suction side is larger at 5 percent of the period, which is the main reason why the lift in this position is lower than that at 95 percent of the period. The top right and bottom left subplots refer instead to the positions at 30 and 70 percent of the period respectively. Their 

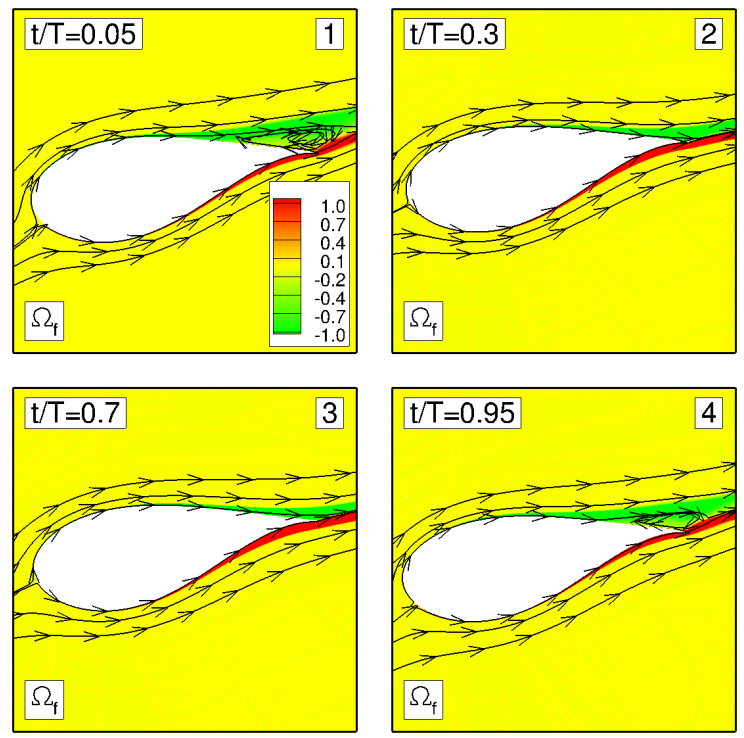

Figure 3: HAWT blade section test case: snapshots of vorticity contours and streamlines at four positions labeled 1 to 4 in Fig. 2 computed with TD 128 simulation. Top left: $5 \%$ of the period; top right: 30 of the \% period; bottom left: $70 \%$ of the period; bottom right: 95 $\%$ of the period.

comparison reveals that in the former position the amount of vorticity on the rear portion of the airfoil pressure side is smaller than in the latter position. This is due to higher velocity of the air stream when the airfoil is at 30 percent of the period, and it results in a thinner boundary layer and a consequently higher viscous stress at the wall. This is the main reason for the higher drag in most of the first half of the period.

To provide further insight into the main characteristics of the hysteretic phenomena discussed above, the static pressure coefficient $c_{p}$ and the skin friction coefficient $c_{f}$ at the four selected positions of the period are examined in Fig. [4 The definitions of $c_{p}$ and $c_{f}$ are respectively:

$$
c_{p}=\frac{p_{w}-p_{\infty}}{0.5 \rho_{\infty}\left(W_{\infty}^{r}\right)^{2}}
$$




$$
c_{f}=\frac{\left|\tau_{w}\right|}{0.5 \rho_{\infty}\left(W_{\infty}^{r}\right)^{2}}
$$

in which the symbols $p_{w}$ and $\tau_{w}$ denote respectively the static pressure and the viscous stress at the airfoil surface. The left subplot of Fig. 4 compares the $c_{p}$ profiles along the airfoil chord at 5 and 95 percent of the period. In the chord is higher than in the latter position. This is a consequence of the higher air velocity in this airfoil area in the first half of the period.

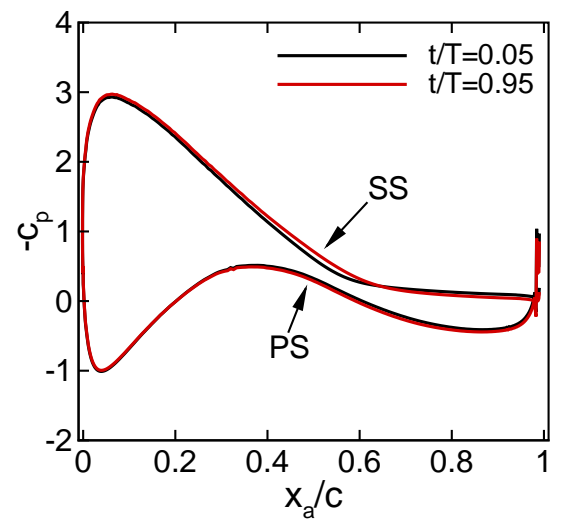

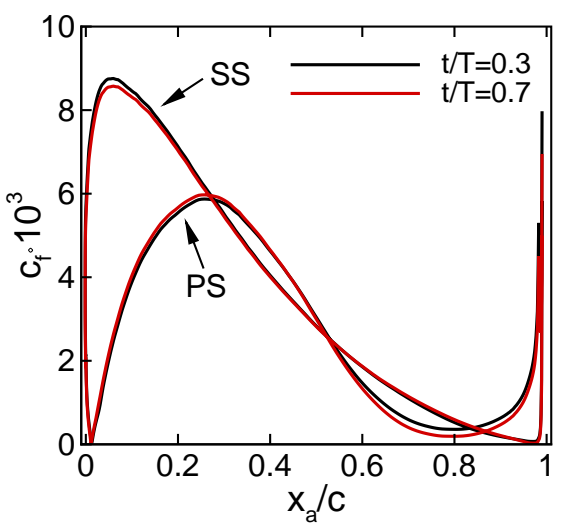

Figure 4: HAWT blade section test case. Left: static pressure coefficient at 5 and 95 percent of the period; right: skin friction coefficient at 30 and 70 percent of the period. All profiles refer to $T D 128$ simulation.

To determine the minimum number of harmonics required to resolve the time-dependent problem at hand with the $H B$ solver achieving a time resolution comparable to that of the $T D 128$ simulation, five $H B$ simulations have been performed. Such simulations use values of $N_{H}$ of $1,2,3,4$ and 5 , and are denoted by the acronym $H B$ followed by the value of $N_{H}$. The hysteretic cycles 
of the $C_{x}, C_{y}$ and $C_{m}^{\prime}$ force coefficients computed by the five $H B$ analyses are plotted against $\phi_{\infty}^{r}$ in the three subplots of Fig. 5 . One notes that using four complex harmonics is sufficient to achieve a resolution of the force coefficients fairly similar to that of the $T D 128$ simulation, as highlighted by the closeness of the TD 128 and the $H B \quad 4$ solutions. It is also observed that a complete reconstruction of the flow unsteadiness by means of the $H B$ solver requires five complex harmonics, as underlined by the fact that the $T D 128$ and the $H B \quad 5$ hysteretic loops are superimposed.

The noticeable size of the hysteresis loops of Fig. 5 also highlights that the level of nonlinearity of the periodic flow field caused by the yawed wind condition requires the use of nonlinear CFD. The use of linear CFD is likely to yield insufficiently accurate estimates of the time-dependent loads required for reliable fatigue and aeroelastic analysis and design of HAWT blades. The $C_{x}$ and $C_{y}$ loops highlight a periodic variation of the contribution of this section to the rotor torque and thrust of about $\pm 22 \%$ and \pm 12 respectively. The variation of the $C_{m}^{\prime}$ coefficient with respect to its mean value is about $\pm 52 \%$, pointing to significant contributions to the blade torsional loads caused by the yawed wind regime. The angles $\phi_{\infty}^{r}$ and $\alpha_{\infty}^{r}$ take their maximum when the blade is vertical and the blade velocity and the yawed wind velocity component have opposite direction, whereas they take their minimum when the blade is vertical and the blade velocity and the yawed wind velocity component have the same direction. Therefore, Fig. 5 highlights that the maximum of all three components of the 600 aerodynamic load occurs when the blade moves in the direction of the yawed wind component, whereas the minimum occurs when the blade moves against the yawed wind component.

It should be noted that the aerodynamic analyses reported above differ significantly from those reported in [11] for the same operating conditions. This 605 is because that paper used the DU91 W2 250LM airfoil, which has a maximum thickness-to-chord ratio of 25 percent. The significantly thicker airfoil used in the present study is more representative of the inboard sections of utility-scale HAWTs, and it also results in higher levels of unsteady flow nonlinearity, a fea- 

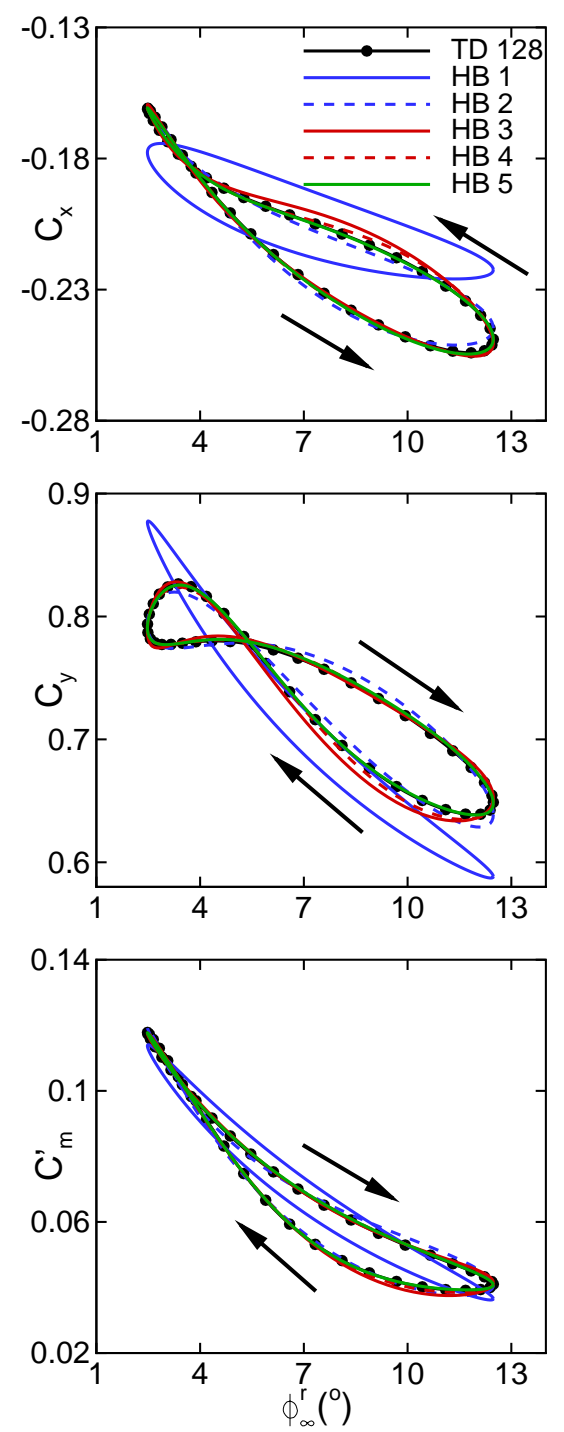

Figure 5: HAWT blade section test case: hysteretic loops of constant head force coefficients computed with five $H B$ simulations and $T D 128$ simulation. Top: horizontal force coefficient; middle: vertical force coefficient; bottom: pitching moment coefficient.

ture that poses higher computational challenges to the $H B$ RANS technology. 


\subsection{Computational performance of the $H B$ solver}

All $H B$ analyses have been run for 20,000 MG cycles, since this was the minimum value required for the convergence of all harmonics of all the force components of these five $H B$ analyses. Each physical time-step of the TD 128 analysis has instead used 2,000 MG iterations, as this value was that required for the convergence of all force components. In order to reduce the periodicity error below the $0.1 \%$ threshold defined at the beginning of the previous subsection, six revolutions had to be simulated starting from a freestream initial condition. For both the HAWT blade section considered in this study and that analyzed in [1]], it has been observed that the number of MG cycles required for the convergence of all harmonics of all the force components is fairly independent of $N_{H}$. The reasons why this number is 20,000 in the present study, and 14,000 in [11] is not only that the unsteady aerodynamics of the problem considered herein is more complex, but also that significantly different multigrid parameters were adopted in the two studies. Here all $H B, T D$ and steady HAWT simulations used 3 smoothing cycles on the fine and medium grids, and 2 smoothing cycles on the coarse grid; all simulations of in 11] used instead 5 smoothing cycles on the fine and medium grids, and 2 smoothing cycles on the coarse grid.

The residual convergence histories of the five $H B$ analyses over the first 8, $000 \mathrm{MG}$ cycles, and the mean residual convergence history of the last period

so of the TD 128 simulation are reported in Fig. 6. The variable on the $x$-axis is the number of MG cycles. For the $H B$ analyses, the variable $\Delta l_{r}$ on the $y$-axis is the logarithm in base 10 of the normalized RMS of all cell-residuals of the four RANS equations of the $2 N_{H}+1$ snapshots. For the TD 128 analysis, the variable $\Delta l_{r}$ on the $y$-axis is instead the logarithm in base 10 of the RMS of all cell-residuals of the four RANS equations of the 128 physical times of the last period. For both $T D$ and $H B$ simulations, each residual history curve is normalized by the RMS value at the first MG cycle. An interesting feature is that the convergence histories of all $H B$ analyses are fairly close to each

other. Some more noticeable differences only exist between the $H B 1$ curve on one hand, and the other four $H B$ curves on the other. This occurrence 
points to the fact that the periodic flow nonlinearity is dominated by the first two harmonics: the contribution of the progressively smaller higher-frequency harmonics of the $H B 3, H B 4$ and $H B 5$ analyses does not affect significantly the spectrum of the linearized operator associated with the integration of these $H B$ set-ups with respect to that associated with the $H B 2$ set-up. The dominance of the first two harmonics in the Fourier reconstruction of this periodic flow is also confirmed by the $H B$ hysteretic force loops of the subplots of Fig. 5 . Inspection of these curves reveals that the largest differences among the $H B$ results are those between the $H B 1$ simulation on one hand and the other four $H B$ simulations on the other. This highlights a significant contribution of the second harmonic to the periodic flow, and rapidly decreasing contributions of the higher order harmonics. Figure 6 also reports the convergence history of the steady problem obtained from the $H B$ set-up by only turning-off the grid motion. The curve of the steady residual history does not differ substantially from those of the $H B$ analyses, and this provides further indication that the level of flow unsteadiness in the problem at hand is moderate.

When using the $H B$ FERK MG smoother given by Eqn. (16) to solve the $H B$ RANS and SST equations, the CPU-time of one $H B$ MG iteration increases in a moderately superlinear fashion with $N_{H}$. This implies that, for a given number of computer cores used for the simulation, the runtime of a $H B N_{H}$ simulation with a given number of MG cycles is higher than $\left(2 N_{H}+1\right)$ times the runtime of the steady simulation using the same number of $M G$ cycles. This overhead is due to the calculation of the $H B$ source term $\Omega V_{H} D \mathbf{Q}_{H}$ appearing in Eqn. (15), and is proportional to $\left(2 N_{H}+1\right)^{2}$. Such an overhead can be quantified by taking the ratio of the measured CPU-time of one $\mathrm{MG}$ iteration of the $H B N_{H}$ analysis and that of one MG cycle of the steady analysis, and dividing such a ratio by $\left(2 N_{H}+1\right)$. The variable $C_{M G}$ thus obtained is reported in the second row of Table 1. It is seen that the overhead for the calculation of the $H B$ source term with the $H B \quad 5$ analysis makes the average CPU-time of one $H B$ MG cycle about 7 percent higher than that of one steady MG cycle.

The $H B$ speed-up parameter, defined as the ratio of the runtime of the 
$T D 128$ simulation and the $H B$ analysis for the five values of $N_{H}$, is reported in the third row of Table 1 It is seen that the $H B \quad 4$ simulation, which yields a very good estimate of the time-dependent loads, reduces the analysis runtime by a factor 8 with respect to the fully time-resolved $T D 128$ analysis. The $H B 5$ analysis, yielding the same resolution of the $T D 128$ analysis reduces the analysis runtime by a factor 6.5 , which is still a remarkable benefit for practical applications.

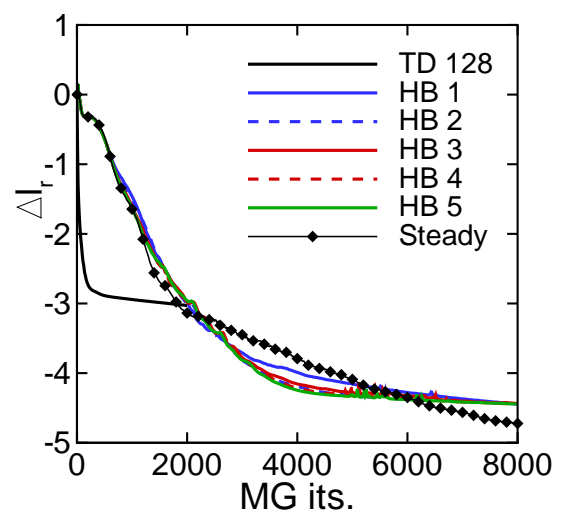

Figure 6: HAWT blade section test case: residual convergence histories of steady, TD and $H B$ solvers.

Table 1: HAWT blade section test case: overhead parameter $C_{M G}$ of $H B$ MG cycle with respect to steady MG cycle, and speed-up of $H B$ analyses with respect to $T D 128$ analysis.

\begin{tabular}{|c|c|c|c|c|c|c|c|}
\hline & $H B 1$ & $H B 2$ & $H B 3$ & $H B 4$ & $H B 5$ & $T D 128$ & steady \\
\hline$C_{M G}$ & 1.038 & 1.044 & 1.056 & 1.066 & 1.073 & - & 1.00 \\
\hline speed-up & 24.7 & 14.7 & 10.4 & 8.0 & 6.5 & 1.0 & - \\
\hline
\end{tabular}

\section{H-Darrieus rotor section}

Here the periodic flow of a H-Darrieus wind turbine is considered. The blade airfoils of this turbine are stacked along straight lines parallel to the tur- 
bine rotational axis. Away from the blade tips, the flow can be considered two-dimensional. The considered rotor has a radius $R_{D}$ of $515 \mathrm{~mm}$, and its 3 blades feature the NACA0021 airfoil with a chord of $85.8 \mathrm{~mm}$. The blade/spoke attachment is at $25 \%$ chord from the airfoil leading edge. The analyzed operating condition is characterized by a freestream velocity $W_{\infty}$ of $9 \mathrm{~m} / \mathrm{s}$, and a rotational speed of 550 RPM. Using standard thermodynamic conditions and the rotor circumferential speed as reference velocity, the Reynolds number based on the airfoil chord is $1.7 \times 10^{5}$; the Mach number associated with the circumferential speed of the rotor is 0.087 . This case study was first reported and analyzed in [43] and [44], and later in several other studies, including [45].

VAWT rotor flows are inherently unsteady because the freestream conditions perceived by each blade vary periodically with frequency determined by the rotor angular speed. Starting by temporarily neglecting the fact that the absolute velocity decreases across the rotor due to the energy transfered from the fluid to the turbine, the modulus of the relative wind velocity $\underline{W}_{\infty}^{r}$ at the rotor radius $R_{D}$, and the angle $\phi_{\infty}^{r}$ between $W_{\infty}^{r}$ and the time-dependent position of the airfoil chord are respectively:

$$
\begin{gathered}
W_{\infty}^{r}=W_{\infty} \sqrt{1+2 \lambda_{D} \cos \theta+\lambda_{D}^{2}} \\
\phi_{\infty}^{r}=\arctan \left(\frac{\sin \theta}{\lambda_{D}+\cos \theta}\right)
\end{gathered}
$$

Here $\lambda_{D}=\Omega R_{D} / W_{\infty}$ is the so-called tip-speed ratio, and the angle $\theta$ defines the azimuthal position of the reference blade. The reference blade has $\theta=0$ when the directions of the absolute velocity $W_{\infty}$ and the entrainment velocity $\Omega R_{D}$ are equal and opposite. The periodic profiles of $M_{\infty}^{r}$, the Mach number associated with $W_{\infty}^{r}$, and $\phi_{\infty}^{r}$ are reported in Fig. 7 These profiles have been computed using $\lambda_{D}=3.3$, which is the tip-speed ratio corresponding to the operating conditions provided above. This value corresponds to near maximum power operation, and unless otherwise stated, all results presented below refer to this value of $\lambda_{D}$. Both curves of Fig. [7] are plotted with a solid line for $0<\theta<$ $180^{\circ}$, the interval corresponding to the reference blade traveling in the upwind 

corresponding to the reference blade traveling in the downwind region of the rotor. This distinction is highlighted because Eqns. (25) and (26) assume that the absolute velocity $W_{\infty}$ is constant throughout the rotor. This is an acceptable approximation in the upwind region but is unacceptable in the downwind region. This is because the energy transfer occurring in the upwind region results in a reduction of the absolute velocity, yielding in turn a significant reduction of both $W_{\infty}^{r}$ and $\phi_{\infty}^{r}$ in the downwind region. This phenomenon is important for the discussion of the rotor torque periodic profile reported below.

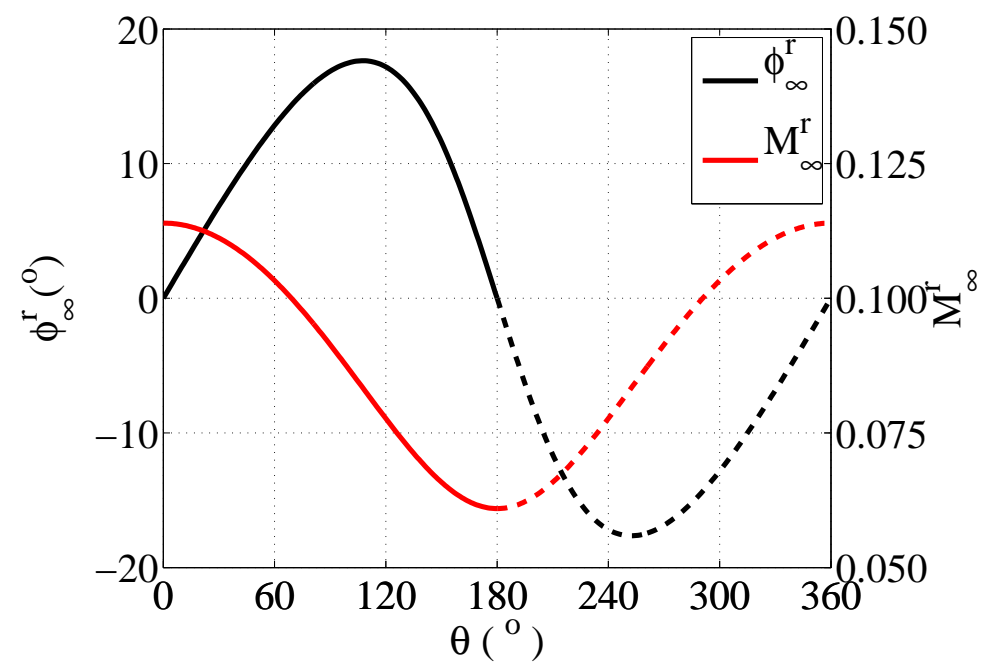

Figure 7: H-Darrieus rotor section test case: theoretical profile of relative flow angle and Mach number against azimuthal position $\theta$ of reference blade.

The physical domain containing the rotor section and its surroundings is delimited by a far field boundary centered at the rotor axis, and is discretized by a structured multi-block grid. The grid is highly clustered in the region around and between the blades, has 729,600 quadrilateral cells and is made up of two subdomains: the circular region of radius $7 R_{D}$ containing the three blades and consisting of 522,240 cells, and the annular region with inner radius 
448 cells around each airfoil, and a distance of the first grid line off the airfoil surface from the airfoil itself of $10^{-5} c$. Enlarged views of the grid around the rotor and the airfoil leading edge areas are reported respectively in the left and right images of Fig. 8 ,
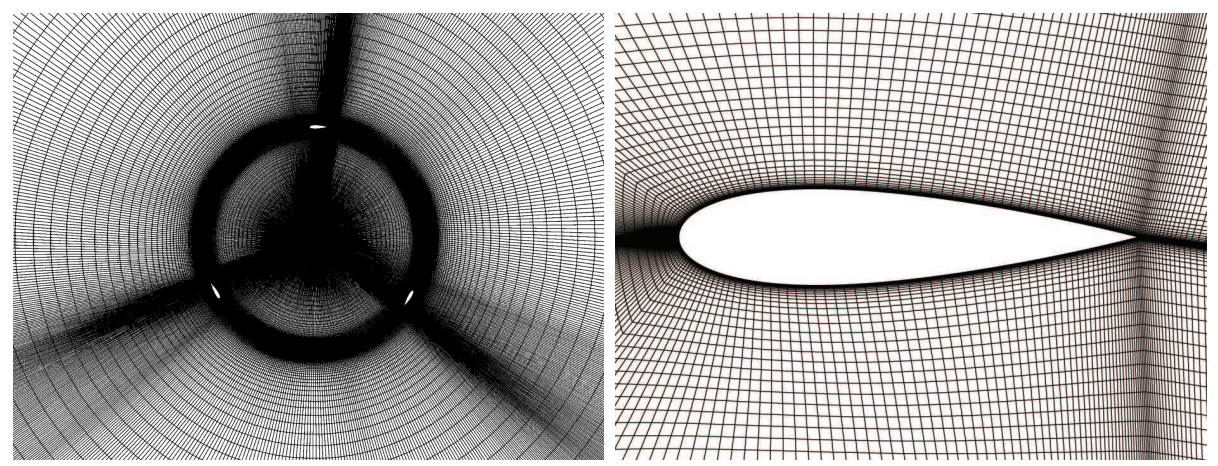

Figure 8: H-Darrieus rotor section test case. Left: grid view in rotor region; right: grid view in leading edge region. (In both cases, only every second line in both directions is plotted).

The identification of two distinct subdomains is irrelevant for the COSA analyses since the entire grid moves with the rotor. The circular interface between the two subdomains was introduced to also enable the simulation of this rotor flow with the commercial ANSYS FLUENT CFD code using the same grid of COSA. FLUENT uses a rotating and a stationary domain and requires a circular sliding interface, which was set to be the circle at distance $7 R_{D}$ from the rotor center. The FLUENT results presented below are obtained with the coupled pressure-based solver [46]. The time-domain simulation of the same rotor flow with both codes has been performed to provide further verification evidence of the predictive capabilities of COSA. All COSA and FLUENT simulations do not use transition modeling and are fully turbulent. In all cases, the far field values of $k$ and $\omega$ are determined by considering a turbulence intensity of 5 percent and a characteristic turbulence length of $70 \mathrm{~mm}$.

All COSA $T D$ and $H B$ simulations discussed below have been performed using the MG solver with 3 grid levels. In all $T D$ simulations reported below, no 
CFL ramping has been used in all $H B$ simulations, and the final CFL number has been set to 2 . The fact that the maximum CFL number of the $T D$ and $H B$ simulations of this problem are different is not surprising. This is because the numerical operators associated with the iterative solution of the $H B$ and $T D$ equations are different, and feature, in general, a different spectral radius. This variable is one of the main parameters determining the maximum pseudo-time step of the iterative solution process and, thus, its highest possible CFL number and convergence rate.

\subsection{Aerodynamic analyses}

The grid described above is used to determine the periodic flow of the considered H-Darrieus rotor. Mesh refinement tests carried out using COSA with the grid under consideration and a finer one with twice as many grid lines in both directions, have highlighted that the present grid with 729,600 cells gives a mesh-independent solution. To determine the minimal time-resolution of the COSA TD analysis required to obtain a solution independent of further reductions of the physical time-step, four different $T D$ simulations have been performed using a number of physical time-steps per period $N_{p}$ of 1440,720 , 360, and 180. In the discussion below, these simulations are denoted by $T D N_{p}$. The starting point of each revolution is the position in which the velocity of the reference blade and the absolute velocity of the wind are parallel and opposite $\left(\theta=0^{\circ}\right)$. From here this blade describes a $180^{\circ}$-circular arc trajectory traveling in the upwind region of the rotor. At the end of this phase $\left(\theta=180^{\circ}\right)$, the blade velocity and the absolute velocity of the wind are parallel and have the same orientation. Thereafter the reference blade travels back to the initial position

$\left(\theta=0^{\circ}\right)$ along the $180^{\circ}$-circular arc trajectory in the downwind region of the rotor.

The output variable used to monitor the convergence of the $T D$ simulations 
to a periodic state is the torque coefficient $C_{T}$ per unit blade length, defined as:

$$
C_{T}=\frac{T_{D}}{\frac{1}{2} \rho_{\infty} W_{\infty}^{2} 2 R_{D}^{2}}
$$

where $T_{D}$ is the torque acting on the reference blade. All four $T D$ simulations 775 have been run until the maximum $C_{T}$ difference at all corresponding positions of two consecutive revolutions became less than $0.2 \%$ of their mean value over the latter period of the cycle pair. The $C_{T}$ profiles of the reference blade over one rotor revolution computed by the four $T D$ analyses are plotted against the azimuthal position $\theta$ of the same blade in Fig. 9. These results show that at least 720 intervals per period are required to achieve a torque prediction independent of further increments of the time resolution. The TD 720 simulation is therefore taken as the reference $T D$ result. Figure 9 also reports the $C_{T}$ profile computed by FLUENT using 900 intervals per period. An excellent agreement between the prediction of the two codes is observed. The two enlarged views of Fig. 9 highlight that some very small differences between the COSA TD 720 and the FLUENT TD 900 predictions only exist around the positions $\theta=90^{\circ}$ and $\theta=220^{\circ}$. Several potential causes of these small differences, such as insufficient space- and/or time- resolutions of either simulation, or lack of low-speed preconditioning [47] in the simulations of the density-based COSA code, have been examined and ruled out. Possible remaining factors accounting for these small differences include a slightly different numerical implementation of the turbulence model. This type of factor, unfortunately, cannot be easily examined due to unavailability of the source code of commercial software. The COSA and FLUENT solutions, however, are extremely close, as also underlined by the fact that the mean torque predicted by the two codes differ by less than 0.15 percent. This high level of agreement constitues a new successful verification test of the COSA code for complex turbulent unsteady flow problems.

The blade torque $T_{D}$ depends largely on the tangential components of the lift and drag forces acting on the blade, and both forces vary significantly during the revolution, because both the relative AoA and the modulus of the relative velocity, the flow velocity perceived by the blade, vary with $\theta$. When the ref- 


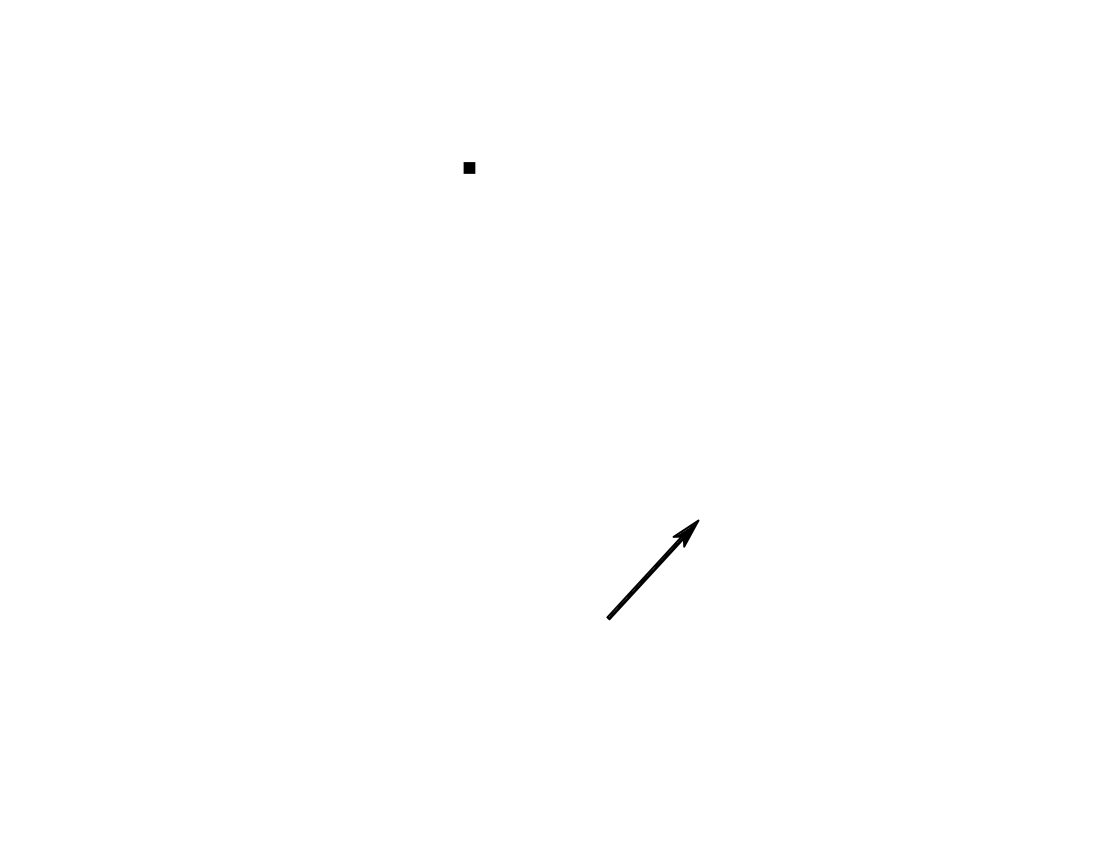

Figure 9: H-Darrieus rotor section test case: periodic profiles of torque coefficient of reference blade against azimuthal position $\theta$ computed with four COSA $T D$ simulations, and FLUENT TD 900 simulation.

erence blade travels in the upwind region of the rotor $\left(0^{\circ}<\theta<180^{\circ}\right)$, a good qualitative estimate of the variation of the relative velocity and the AoA is provided by the $M_{\infty}^{r}$ and $\phi_{\infty}^{r}$ curves of Fig. 7 This figure shows that $\phi_{\infty}^{r}$ achieves its maximum at $\theta \approx 90^{\circ}$. This corresponds to maximum lift coefficient of the airfoil. The peak of the torque coefficient of Fig. 9 at this azimuthal position is due to the high value of the tangential projection of the lift force. In the downwind region of the rotor, however, the absolute velocity decreases considerably with respect to its initial value $W_{\infty}$, and this results in significantly lower values of the AoA in this region. This is the reason why the torque for $180^{\circ}<\theta<360^{\circ}$ does not experience the high values and the peak observed in the first half of the period.

To discuss the main aerodynamic phenomena occurring at this operating regime, assess in further detail the differences between the COSA and FLUENT 
time step of the simulation, the blade profiles of static pressure coefficient $c_{p}$ and skin friction coefficient $c_{f}$ at $\theta=0^{\circ}, \theta=99^{\circ}$, and $\theta=240^{\circ}$ are analyzed. The definitions of $c_{p}$ and $c_{f}$ are given respectively by Eqn. (23) and Eqn. (24). The top subplot row of Fig. 10 compares the $c_{p}$ profiles of the COSA TD 360 and positions indicated above, whereas the $c_{f}$ profiles for the same simulations and azimuthal positions are provided in the bottom subplot row. In all subplots the variable $x_{a} / c$ along the $\mathrm{x}$-axis is the axial position along the airfoil normalized by the chord.

The effect of the rapid increment of the AoA from its low value at $\theta=0^{\circ}$ to its highest levels shortly before $\theta=99^{\circ}$ is visible in the substantial loading increment between these two azimuthal positions (top left and middle subplots). Here the area between the suction side and pressure side branches of $c_{p}$ is taken as a measure of the aerodynamic loading. At $\theta=99^{\circ}$, the flow on the airfoil $c_{f}$ cusp at about $50 \%$ chord (bottom middle subplot). At $\theta=240^{\circ}$ the airfoil loading is fairly low (top right subplot) due to the reduction of the absolute velocity caused by the energy extraction from the fluid occurring in the upwind region of the rotor. The reduction of the absolute velocity results in a significant can be found in [45].

From a numerical viewpoint, one sees that the largest difference between the COSA TD360 and TD720 simulations occurs at $\theta=99^{\circ}$, a result consistent with the differences between these two simulations observed in the torque coefficient predictions. All subplots also confirm that the overall agreement between the COSA TD 720 and and FLUENT TD 900 simulations is excellent. Fig. 10. shows that some small differences only occur in the initial part of the $c_{f}$ profiles, most notably at $\theta=99^{\circ}$ and $\theta=240^{\circ}$. As reported above, these differences may be due to slightly different numerical implementation of the turbulence model 845 in the two codes. 

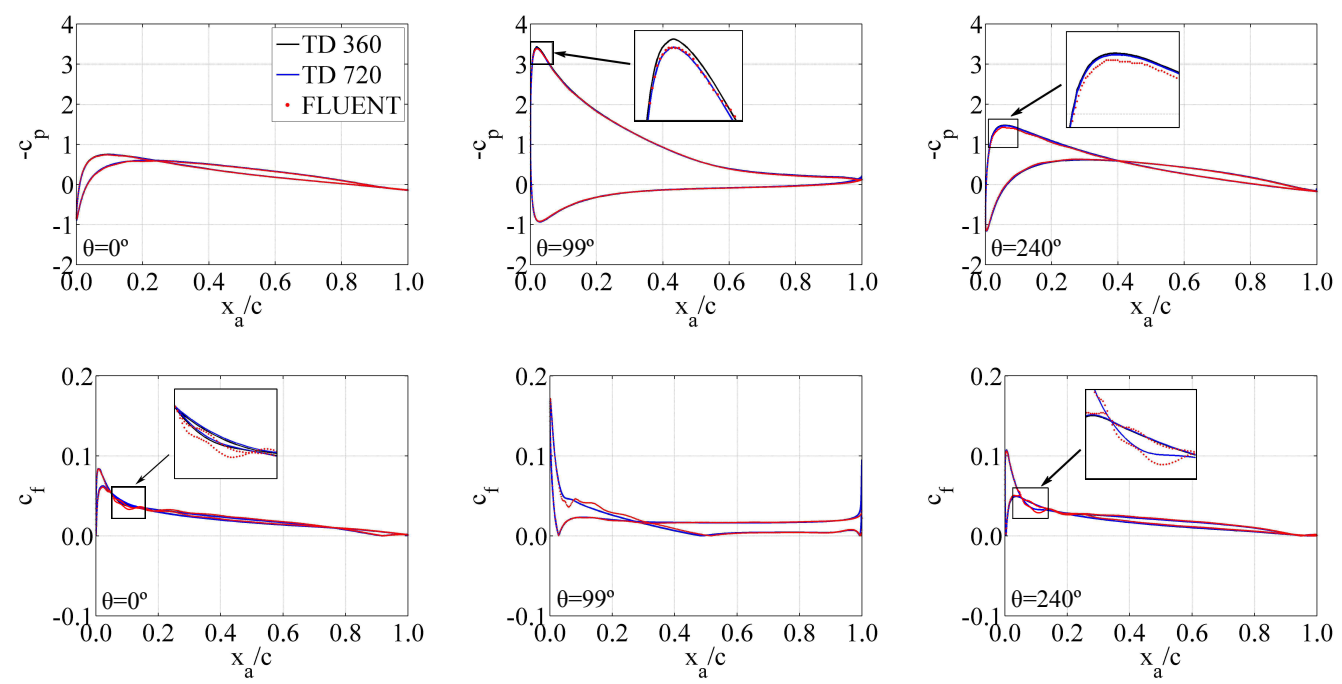

Figure 10: H-Darrieus rotor section test case: airfoil static pressure coefficient $\left(c_{p}\right)$ and skin friction coefficient $\left(c_{f}\right)$ of reference blade at three azimuthal positions $\theta$ computed with COSA TD 360 and TD 720 simulations, and FLUENT TD 900 simulation. Left: $c_{p}$ (top) and $c_{f}$ (bottom) at $\theta=0^{\circ}$; middle: $c_{p}$ (top) and $c_{f}$ (bottom) at $\theta=99^{\circ}$; right: $c_{p}$ (top) and $c_{f}$ (bottom) at $\theta=240^{\circ}$.

The high level of stall associated with the highlighted flow separation at $\theta=$ $99^{\circ}$ is clearly visible in Fig. 11, which provides streamlines and Mach contours in the trailing edge region obtained with the COSA TD 720 simulation (left) and the FLUENT TD 900 simulation (right). Once more, an excellent agreement between the two predictions is observed.

COSA and FLUENT time-domain simulations of the H-Darrieus rotor flow considered above were also carried out in [45], but the agreement between the analyses of the two codes highlighted above is significantly better than that observed in [45]. This is because the FLUENT simulations of both studies used a limiter of the $k$ and $\omega$ production terms similar to that of Eqn. (14) with $l_{k}=10$ (this is a default setting of FLUENT), whereas no limiter of the $k$ and $\omega$ production terms was used for the COSA simulations in [45]. On the other hand, the limiter of Eqn. (14) with $l_{k}=10$ has also been used for the 

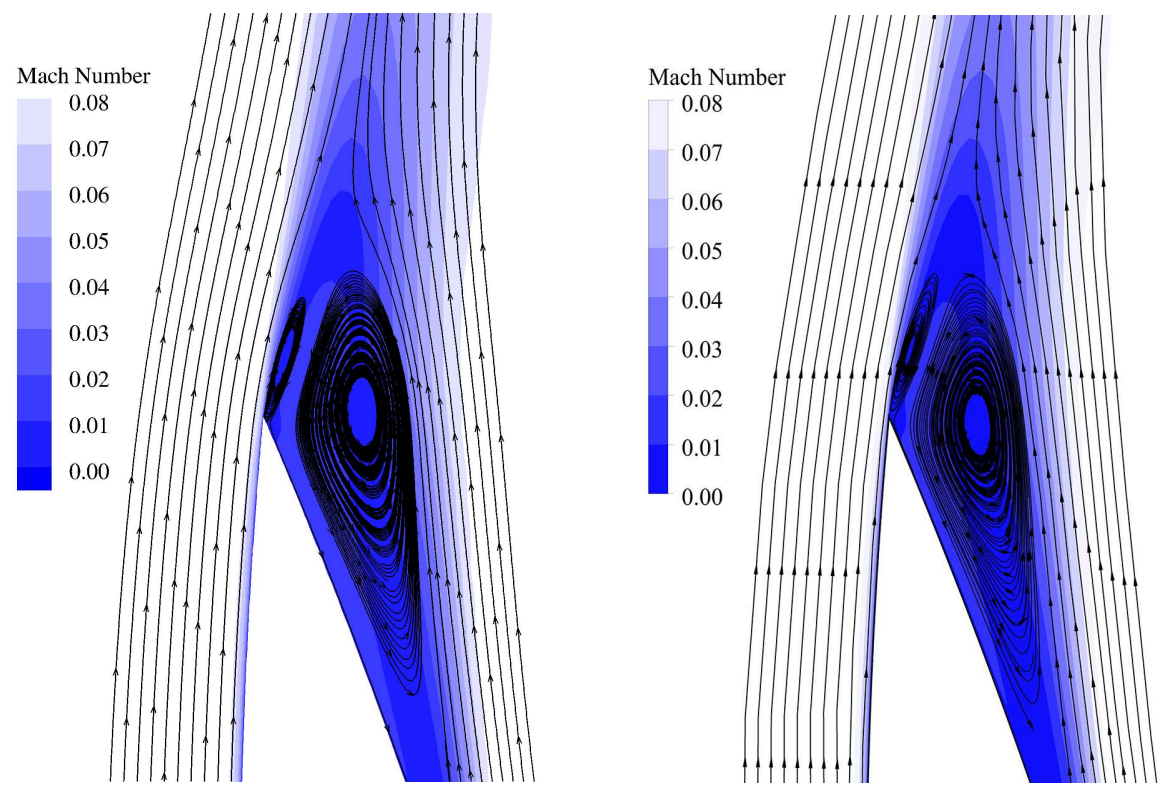

Figure 11: H-Darrieus rotor section test case: Mach contours and streamlines in reference blade trailing edge region at azimuthal position $\theta=99^{\circ}$ computed with COSA TD 720 simulation (left) and FLUENT TD 900 simulation (right).

new COSA VAWT analyses reported herein. The significant improvement of the agreement between the predictions of the two codes emphasizes the high solution sensitivity to predominantly numerical features of complex simulation systems.

To investigate the possibility of more efficiently solving this periodic VAWT flow problem with the $H B$ solver and assess the level of accuracy achievable by using this approach rather than the standard $T D$ method, this $\lambda_{D}=3.3$ VAWT flow field has been solved with three $H B$ simulations. Such simulations use values of $N_{H}$ of 16,32 and 64 . The periodic profiles of the torque coefficient $C_{T}$ computed by these three $H B$ analyses and the $T D 720$ simulation are plotted against $\theta$ in the left subplot of Fig. 12. One notes that the $H B \quad 32$ and the $H B \quad 64$ profiles are fairly close to each other, indicating that most of the flow periodic unsteadiness resolved by the $H B$ analysis is contained in the first 32 Fourier modes. However, there exist some differences between these two $H B$ 
results and the reference $T D 720$ solution: unlike the $T D$ profile, both of these $H B$ profiles have some oscillations for $90^{\circ}<\theta<180^{\circ}$, and the $H B$ profiles also appear to slightly underpredict the torque for $180^{\circ}<\theta<240^{\circ}$. The primary reason for these discrepancies between the $T D$ and the $H B$ solutions is likely to be that the residuals of the $H B$ flow snapshots featuring the highest values of AoA, where the flow is significantly stalled, experience premature stagnation ending in a limit cycle and preventing the $H B$ simulation from fully converging. Such premature stagnation of the residuals is a consequence of the stall induced by the high AoA. Since the high-dimensional $H B$ method solves the frequencydomain governing equations as a set of coupled steady problems, the premature residual stagnation of the steady problems associated with the highest values of AoA ending in a limit cycle prevents the full convergence of the entire set 885 of equations. This issue has also been reported in the dynamic stall analyses of [27]. The oscillations of the $H B$ torque profiles for $90^{\circ}<\theta<180^{\circ}$ reflect such limit cycles. The right subplot of Fig. 12 compares the $C_{T}$ profiles of the TD 720 and the mean profiles of the $H B 32$ and $H B 64$ simulations. Such mean profiles are obtained by averaging the torque profiles of the last $500 \mathrm{MG}$ cycles of each $H B$ analysis. One notes that the agreement between the $T D$ and the mean $H B$ profiles of the torque coefficient for $90^{\circ}<\theta<180^{\circ}$ is significantly improved, supporting the assumption that the $H B \quad 32$ and $H B \quad 64$ torque coefficient profiles reported in the left subplot of Fig. 12 are just instantiations of a lowlevel limit cycle. For this particular problem, this result is fairly independent of the number of final MG cycles of the $H B$ simulation used to average the $H B$ torque profile as long as this number is 300 or more. This is shown in Fig. 13 , where the percentage difference of the reference blade torque averaged over the last 100,300,500 and $1000 \mathrm{MG}$ cycles of the $H B 32$ simulation and the reference blade torque of the $T D 720$ simulation is plotted against the rotor azimuthal position $\theta$ (for each $\theta$, the torque differences are normalized by the maximum value of the $T D \quad 720$ torque profile). One sees that averaging the $H B \quad 32$ torque profile over $300 \mathrm{MG}$ cycles or more yields the same level of fluctuations with respect to the $T D 720$ estimate and maximum error amplitudes smaller 
than 5 percent. This averaging process is not fully consistent with the physics because the solution process of the $H B$ equations does not correspond to a time-accurate march. However, the RK pseudo-time-marching component of the solution process is expected to qualitatively reflect unsteady flow features. For $\lambda_{D}=3.3$, the averaging process yields a torque profile that differs by less than 5 percent from the reference $T D$ estimate. This error level is likely to be acceptable for preliminary design applications. This aspect is discussed in further detail at subsection 5.3

The interpretation of the oscillations of the $H B$ solutions reported above is in line with the analysis of the flow physics-induced numerical instabilities of a multigrid smoother for the solution of the nonlinear NS equations and their linearized counterpart reported in [48]. It has also been found that the agreement between the $T D$ and the $H B$ simulations improves substantially, becoming comparable to that observed for the HAWT blade section discussed above, as the tip-speed ratio $\lambda_{D}$ increases. This happens because the maximum AoA and the amount of flow stall decrease as $\lambda_{D}$ increases.
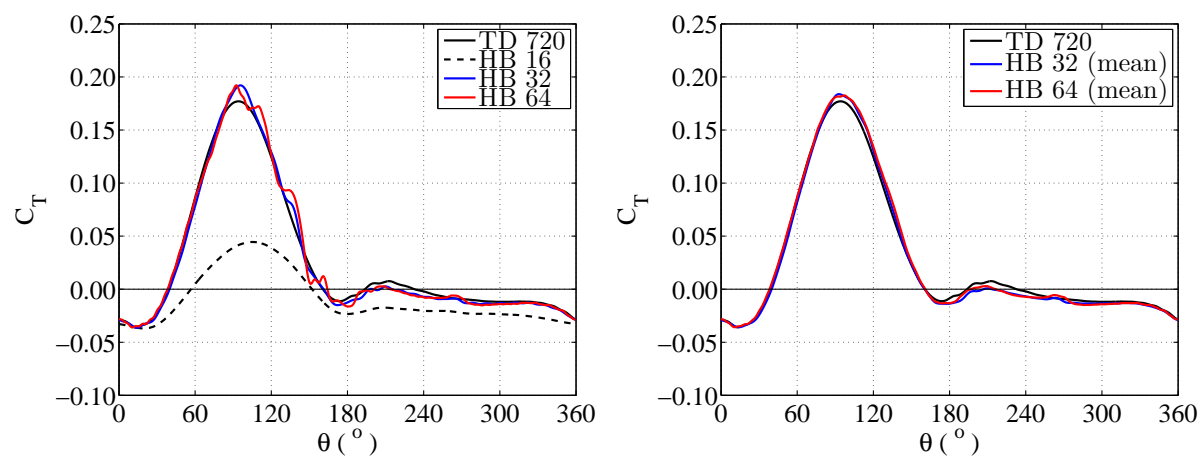

Figure 12: H-Darrieus rotor section test case: periodic profiles of torque coefficient of reference blade against azimuthal position $\theta$ computed with three COSA $H B$ simulations, and COSA $T D 720$ simulation. Left: $H B$ torque profiles at last MG cycle of simulation; right: $H B$ torque profiles averaged over last 500 MG cycles. 


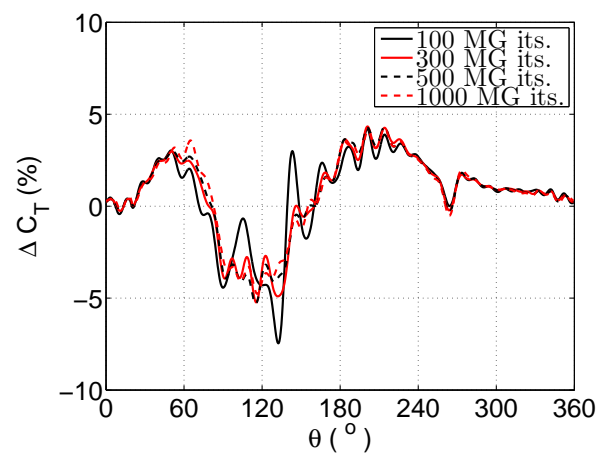

Figure 13: H-Darrieus rotor section test case: percentage difference of reference blade torque averaged over last 100, 300, 500 and 1000 MG cycles of $H B 32$ simulation and reference blade torque of $T D 720$ simulation plotted against rotor azimuthal position.

\subsection{Computational performance of the $H B$ solver}

Each physical time-step of the TD 720 analysis has required $200 \mathrm{MG}$ cycles to achieve a reduction of the RMS of the RANS equations of nearly seven orders. This is highlighted in the left subplot of Fig. 14, which reports the mean convergence history of the last period of the TD 720 simulation. However, it has also been verified that all force components are fully converged at all times of the revolution after just 100 cycles. In order to reduce the periodicity error below the $0.2 \%$ threshold defined at the beginning of the previous subsection, thirty revolutions had to be simulated starting from a freestream initial condition. It has also been verified that this periodicity error threshold is achieved after thirty revolutions with both aforementioned values of the number of MG cycles per physical time.

In the case of the $H B$ simulations, the convergence trends examined above are reversed: it has been observed that stagnation of the $H B$ residuals is achieved long before all force components achieve a constant level. Moreover, the number of $H B$ cycles required to achieve a constant level of all force components has been different for all three $H B$ simulations: the $H B \quad 16, H B 32$ and $H B \quad 64$ have required respectively 15,000, 12,000 and 9,000 MG cycles. The residual convergence histories of the three $H B$ analyses over 12,000 MG 
cycles are reported in the right subplot of Fig. 14. One notes that the mean residuals of the $H B$ simulations decrease by only two orders before stagnating. This is most likely due to the occurrence of a limit cycle in the pseudo-time march process associated with the solution of the $H B$ RANS and SST equations. Indeed, examination of the convergence histories of the force components associated with the 65 flow snapshots of the $H B \quad 32$ simulation shows that the force components corresponding to the positions at which the AoA lies in a small neighborhood of its maximum $\left(90^{\circ}<\theta<130^{\circ}\right)$ present an oscillatory behavior about a mean value, whereas the force components corresponding to all other positions converge to fairly constant values.
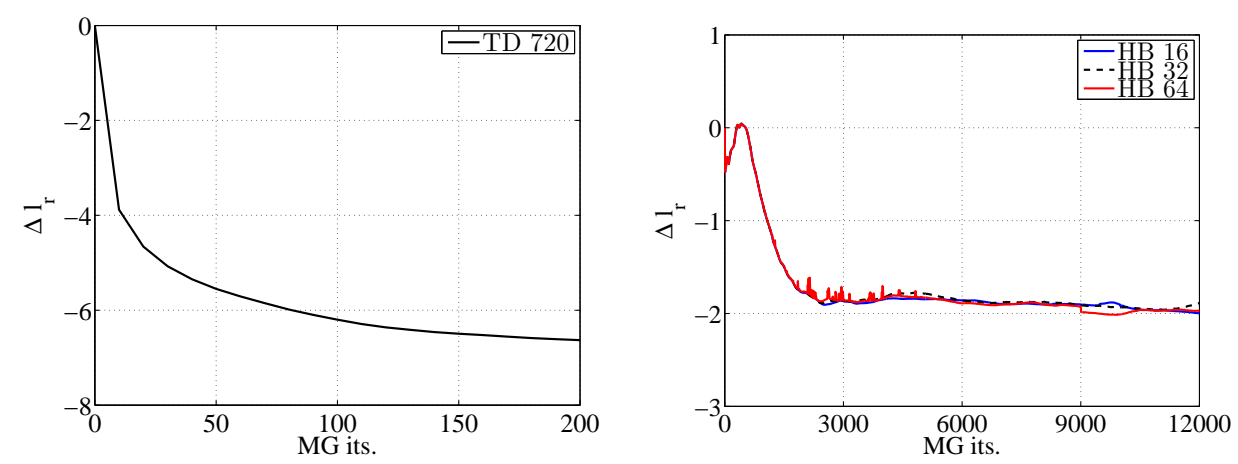

Figure 14: H-Darrieus rotor section test case: residual convergence histories of $T D$ and $H B$ simulations. Left: mean convergence history over last period of $T D 720$ simulation; right: converge histories of three $H B$ simulations.

All TD analyses reported in this section could be performed only using the PIRK smoother [37], since the FERK integration has been found numerically stable only for unacceptably low CFL numbers. Similarly to the HAWT blade section test case, however, all $H B$ analyses reported in this section could be performed with the FERK MG Algorithm (16). Also for the present H-Darrieus rotor section test case the overhead of the FERK $H B$ MG cycle with respect to one steady MG cycle, arising due to the calculation of the $H B$ source term source term $\Omega V_{H} D \mathbf{Q}_{H}$, has been analyzed. The $H B$ overhead variable $C_{M G}$ defined in subsection 4.2 for the three $H B$ simulations discussed above is reported in 
the second row of Table 2. It is seen that the overhead for the calculation of the $H B$ source term with the $H B 32$ analysis makes the average CPU-time of one $H B$ MG cycle 50 percent higher than that of one steady MG cycle; the $H B$ source term overhead of the $H B \quad 64$ analysis makes its MG cycle more than twice as expensive as the steady MG cycle.

The $H B$ speed-up parameter, defined as the ratio of the runtime of the TD 720 simulation using $100 \mathrm{MG}$ cycles per physical time and the $H B$ analysis for the three values of $N_{H}$, is reported in the third row of Table2. It is seen that the $H B 32$ analysis, which brings the closest result to the $T D 720$ simulation is 85 percent faster than the latter analysis.

Table 2: H-Darrieus rotor section test case: overhead parameter $C_{M G}$ of $H B \mathrm{MG}$ cycle with respect to steady MG cycle, and speed-up of $H B$ analyses with respect to $T D 720$ analysis.

\begin{tabular}{|c|c|c|c|c|c|}
\hline & HB 16 & HB 32 & HB 64 & TD 720 & steady \\
\hline$C_{M G}$ & 1.19 & 1.50 & 2.11 & - & 1.0 \\
\hline MG cycles & 15,000 & 12,000 & 9,000 & $2,160,000$ & \\
\hline speed-up & 3.66 & 1.85 & 0.88 & 1.0 & - \\
\hline
\end{tabular}

\subsection{Discussion}

The $H B$ speed-up achievable for the analysis of the H-Darrieus rotor section is significantly lower than that achieved for the analysis of the HAWT blade section. Moreover, due to the substantially higher amount of dynamic stall, the $H B$ analysis of the VAWT problem does not enable one to achieve a solution accuracy comparable with that of the $T D$ solution, unlike what observed for the HAWT problem.

Nevertheless, the mean power output predicted by the $H B \quad 32$ analysis is in relatively good agreement with the TD 720 analysis over a wide range of tip-speed ratios. This is highlighted in Fig. 15, which shows the comparison of the rotor power curve predicted by the $T D$ and the $H B$ simulations for $2.4 \leq \lambda_{D} \leq 4$. The errors of the $H B$ power predictions with respect to the 
may be required to make this technology computationally competitive with very fast low-fidelity methods, such as BEMT codes.

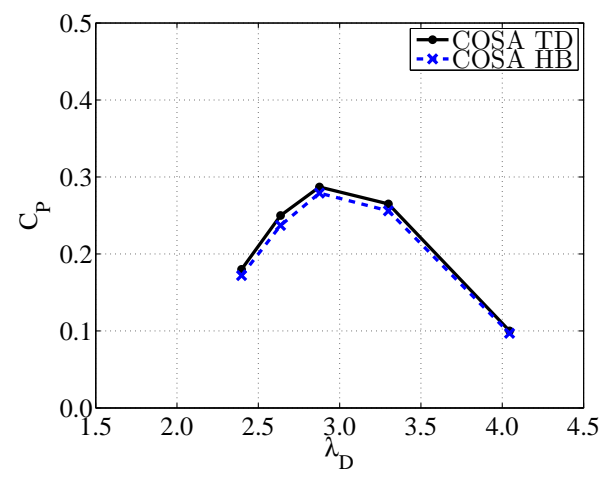

Figure 15: H-Darrieus rotor section test case: nondimensionalized power curves computed with COSA TD 720 and $H B 32$ simulations.

\section{Conclusions}

A detailed assessment of the actual benefits achievable by using a $H B$ RANS CFD code featuring the SST turbulence model for the analysis of wind turbine periodic aerodynamics has been presented. 
Table 3: H-Darrieus rotor section test case: \% difference between nondimensionalized power curves computed with COSA TD 720 and HB 32 simulations.

\begin{tabular}{|c|c|c|c|c|c|}
\hline$\lambda_{D}$ & 2.40 & 2.64 & 2.88 & 3.30 & 4.05 \\
\hline$C_{P}($ TD 720) & 0.180 & 0.250 & 0.287 & 0.265 & 0.100 \\
\hline$C_{P}(H B$ 32) & 0.172 & 0.237 & 0.279 & 0.256 & 0.097 \\
\hline$\Delta C_{P}(\%)$ & 4.44 & 5.20 & 2.79 & 3.40 & 3.00 \\
\hline
\end{tabular}

In the case of utility-scale horizontal axis machines, the assessment was based on the analysis of the periodic flow field past the $30 \%$ blade section of a $164 \mathrm{~m}$ diameter rotor in a $45^{\circ} 13 \mathrm{~m} / \mathrm{s}$ yawed wind. Significant hysteresis cycles of all forces acting on the blade section were observed, with variations of the axial and tangential force components of about $\pm 12 \%$ and $\pm 22 \%$, respectively, of their mean values, and variations of the sectional torque of about $52 \%$ of its mean value. The $H B$ analysis using 4 complex harmonics reproduced the solution of the fully time-resolved $T D 128$ analysis nearly 10 times more rapidly than the TD analysis. The $H B$ RANS method has a strong potential of improving utility-scale HAWT design since it enables the use of the NS equations to determine fatigue-inducing and power-reducing loads more accurately than low-fidelity analysis methods and more rapidly than conventional time-domain NS CFD. The high computational efficiency of the $H B$ technology, possibly with the initial support of reliable reduced order modeling, offers the possibility of optimizing the design of HAWT rotors, accurately accounting for complex unsteady flow features.

For VAWTs the assessment was based on the analysis of the periodic flow of the rotor section of a small H-Darrieus rotor working at a near-maximum power tip-speed ratio of 3.3. Although the overall agreement of the $H B$ and $T D$ analyses was fairly good, the comparison of the torque profiles and the power coefficient of the two simulations revealed differences of up to 5 percent. This is due to the high level of stall characterizing the operation of Darrieus 
rotors at and around peak power conditions which prevents the pseudo-timemarching solution of the $H B$ equations from fully converging. This flow regime type is quite different from that typically encountered in utility-scale HAWT rotors, which experience much smaller stall levels due to effective rotor speed and blade pitch control systems, and whose $H B$ periodic flow analyses thus present fewer numerical difficulties. Nevertheless, the $H B$ and $T D$ VAWT rotor predictions are sufficiently close to consider future use of the $H B$ method for VAWT preliminary design.

For aerodynamic problems charactereized by high stall levels, like Darrieus rotor flows, more research aiming at alleviating the numerical instabilities of the $H B$ solver and improving its convergence properties appears to be needed. Code stabilization techniques previously used to remove this type of instability, such as the Recursive Projection Method [49] and the Proper Orthogonal Decomposition [50], could be tested also for improving the $H B$ NS technology.

A novel fully coupled MG solution procedure of the compressible RANS and SST turbulence model equations that uses a point-implicit integration of the turbulence equations has been discussed. An important approximation to the integration of the SST equations, valid for low-speed flows, resulting in a partial decoupling of the two SST equations, and yielding higher numerical stability of both steady and $H B$ equations, has also been discussed.

Finally, it is noted that the runtime of $H B$ NS solvers can be substantially further reduced by exploiting the possibility of parallelizing the routine cycles of the $H B$ code looping over the $2 N_{H}+1$ flow snapshots [51, 39]. This can be viewed as an effective approach to time-parallelizing the solution of periodic flows, an opportunity unavailable in this form in $T D$ codes.

\section{Acknowledgement}

This work made use of the facilities of N8 HPC provided and funded by the N8 consortium and EPSRC (Grant No.EP/K000225/1). The Centre is co-ordinated by the Universities of Leeds and Manchester. The authors also 
acknowledge use of Hartree Centre resources in this work. The STFC Hartree Centre is a collaborative research programme in association with IBM providing High Performance Computing platforms funded by the UK's investment in e-

Infrastructure. Dr. F. Balduzzi, Dr. A. Bianchini and Dr. L. Ferrari are acknowledged for providing the FLUENT-based VAWT results reported in this paper.

\section{References}

[1] M. Hansen, Aerodynamics of wind turbines, Second edition, Earthscan, London, 2008.

[2] J. Manwell, J. McGowan, A. Rogers, Wind Energy Explained. Theory, Design and Application, John Wiley and Sons Ltd., 2002.

[3] S. Tsalicoglou, S. Barber, N. Cholani, R. Abhari, Effect of Flow Inclination on Wind Turbine Performance, Journal of Engineering for Gas Turbine and Power 134 (12) (2012) 122601.1-122601-8.

[4] M. Brahimi, A. Allet, I. Paraschivoiu, Aerodynamic analysis models for vertical-axis wind turbines, International Journal of Rotating Machinery 21 (1) (1995) 15-21.

[5] E. Amet, T. Maitre, C. Pellone, j.-L. Achard, 2D Numerical Simulations of Blade-Vortex Interaction in a Darrieus Turbine, Journal of Fluids Engineering 131 (2009) 111103.1-111103.15.

[6] C. Simao-Ferreira, A. van Zuijlen, H. Bijl, G. van Bussel, G. van Kuik, Simulating dynamic stall on a two-dimensional vertical-axis wind turbine: verification and validationwith particle image velocimetry data, Wind Energy 13 (2010) 1-17.

[7] M. Hansen, J. Sorensen, S. Voutsinas, N. Sorensen, H. Madsen, State of the art in wind turbine aerodynamics and aeroelasticity, Progress in Aerospace Sciences 42 (2006) 285-330. 
[15] C. Tsalicoglou, S. Jafari, S. Chokani, R. Abhari, Rans computations of

[8] P. Jain, Wind Energy Engineering, McGraw-Hill, New York, NY, USA, 2011.

[9] C. Bak, Aerodynamic design of wind turbine rotors, in: W. Gentzsch, U. Harms (Eds.), Advances in wind turbine blade design and materials, Vol. 47 of Energy, Woodhead Publishing, Cambridge, UK, 2013, pp. 59108.

[10] H. Himmelskamp, Profile investigations of a rotating airscrew, in: $\mathrm{PhD}$ Dissertation Goettingen 1945, Report and Translation 832, MAP Voelkenrode, 1947.

[11] M. Campobasso, F. Gigante, J. Drofelnik, Turbulent Unsteady Flow Analysis of Horizontal Axis Wind Turbine Airfoil Aerodynamics Based on the Harmonic Balance Reynolds-Averaged Navier-Stokes Equations, ASME paper GT2014-25559 (June 2014).

[12] H. Madsen, N. Sorensen, S. Schreck, Yaw Aerodynamics Analyzed with Three Codes in Comparison with Experiments, AIAA paper 2003-0519, 41st AIAA Aerospace Sciences Meeting and Exhibit, Reno, Nevada (January 2003).

[13] A. Le Pape, V. Gleize, Improved Navier-Stokes Computations of a Stallregulated Wind Turbine Using Low Mach Number Preconditioning, AIAA paper 2006-1502, 44th AIAA Aerospace Sciences Meeting and Exhibit,

[14] N. Sezer-Uzol, A. Gupta, L. Long, 3D Time-Accurate Inviscid and Viscous cfd Simulations of Wind Turbine Rotor Flow Fields, in: I. Tuncer, U. Guelcat, D. Emerson, K. M. (Eds.) (Eds.), Parallel Computational Fluid Dynamics 2007, Vol. 67 of Lecture Notes in Computational Science and Engineering, Springer-Verlag, 2007, pp. 457-464. 
wind turbine near-wake aerodynamics in uniform and yawed inflow, ASME paper GT2013-96034 (June 2013).

[16] A. Iida, K. Kato, A. Mizuno, Numerical Simulation of Unsteady Flow and Aerodynamics Performance of Vertical Axis Wind Turbines with LSE, AIAA paper 78-257, 16th Australian Fluid Mechanics Conference, Gold Coast, Australia (December 2007).

[17] R. Howell, N. Qin, J. Edwards, N. Durrani, Critical issues in the simulation of darrieus wind turbines, Renewable Energy 35 (2010) 412-422.

[18] A. Rossetti, G. Pavesi, Comparison of different numerical approaches to the study of the h-darrieus turbines start-up, Renewable Energy 50 (2013) $7-19$.

[19] F. Balduzzi, A. Bianchini, R. Maleci, G. Ferrara, L. Ferrari, Critical issues in the simulation of darrieus wind turbines, Renewable Energy 85 (2016) 419-435.

[20] K. Hall, J. Thomas, W. Clark, Computations of Unsteady Nonlinear Flows in Cascades Using a Harmonic Balance Technique, AIAA Journal 40 (5) (2002) 879-886.

[21] E. van der Weide, A. Gopinath, A. Jameson, Turbomachinery Applications with the Time Spectral Method, AIAA paper 2005-4905, 17th AIAA Computational Fluid Dynamics Conference, Torontp, Ontario, Canada (June 2005).

[22] X. Su, X. Yuan, Implicit Solution of Time-Spectral Method for Periodic Unsteady Flows, International Journal for Numerical Methods in Fluids 63 (7) (2010) 860-876.

[23] A. Da Ronch, A. McCracken, K. Badcock, , M. Widhalm, M. Campobasso, Linear Frequency Domain and Harmonic Balance Predictions of Dynamic Derivatives, Journal of Aircraft 50 (3) (2013) 694-707. 
[24] F. Sicot, G. Puigt, M. Montagnac, Block-Jacobi Implicit Algorithms for the Time Spectral Method, AIAA Journal 46 (12) (2008) 3080-3089.

[25] M. A. Woodgate, K. J. Badcock, Implicit Harmonic Balance Solver for Transonic Flows with Forced Motions, AIAA Journal 47 (4) (2009) 893901.

[26] M. Campobasso, M. Baba-Ahmadi, Analysis of Unsteady Flows Past Horizontal Axis Wind Turbine Airfoils Based on Harmonic Balance Compressible Navier-Stokes Equations with Low-Speed Preconditioning, Journal of Turbomachinery 134 (6).

[27] J. Howison, K. Ekici, Unsteady Analysis of Wind Turbine Flows Using the Harmonic Balance Method, AIAA paper 2013-1107, 51st AIAA Aerospace Sciences Meeting including the New Horizons Forum and Aerospace Exposition, Grapewine (Dallas/Ft. Worth Region), Texas (January 2013).

[28] P. Spalart, S. Allmaras, A one-equation turbulence model for aerodynamic flows, La Recherche Aerospatiale 1 (1994) 5-21.

[29] J. Howison, K. Ekici, Dynamic stall analysis using harmonic balance and correlation based $\gamma-r e_{\theta}$ transition models for wind turbine applications, Wind Energy 18 (2015) 2047-2063.

[30] F. Menter, Two-equation Turbulence-models for Engineering Applications, AIAA Journal 32 (8) (1994) 1598-1605.

[31] J. Vassberg, A. Gopinath, A. Jameson, Revisiting the Vertical-Axis WindTurbine Design using Advanced Computational Fluid Dynamics, AIAA paper 2005-47, 43rd AIAA Aerospace Sciences Meeting and Exhibit, Reno, Nevada (January 2005).

[32] D. Wilcox, Reassessment of the Scale-Determining Equation for Advanced Turbulence Models, AIAA Journal 26 (11) (1988) 1299-1310. 
[38] M. Campobasso, J. Drofelnik, Compressible Navier-Stokes analysis of an oscillating wing in a power-extraction regime using efficient low-speed preconditioning, Computers and Fluids 67 (2012) 26-40.

[39] A. Jackson, M. Campobasso, M. Baba-Ahmadi, On the Parallelization of

[33] W. Jones, B. Launder, The Calculation of Low-Reynolds-Number Phenomena with a Two-Equation Model for Turbulence, International Journal of Heat and Mass Transfer 16 (1973) 1119-1130.

[34] F. Liu, X. Zheng, A Strongly Coupled Time-Marching Method for Solving the Navier-Stokes and $K-\omega$ Turbulence Model Equations with Multigrid, Journal of Computational Physics 128 (2) (1996) 289-300.

[35] F. Lin, F. Sotiropoulos, Strongly-Coupled Multigrid Method for 3-D Incompressible Flows Using Near-Wall Turbulence Closures, Journal of Fluids Engineering 119 (1997) 314-324.

[36] L. Liu, J. Thomas, E. Dowell, P. Attar, K. Hall, A comparison of classical and high dimensional harmonic balance approaches for a Duffing oscillator, Journal of Computational Physics 215 (1) (2006) 298-320.

[37] M. Campobasso, A. Piskopakis, J. Drofelnik, A. Jackson, Turbulent NavierStokes Analysis of an Oscillating Wing in a Power-Extraction Regime Using the Shear Stress Transport Turbulence Model, Computers and Fluids 88 (2013) 136-155.

a Harmonic Balance Compressible Navier-Stokes Solver for Wind Turbine Aerodynamics, ASME paper GT2011-45306 (2011).

[40] F. Menter, Zonal Two Equation K- $\omega$ Turbulence Models for Aerodynamic Flows, AIAA paper 93-2906, 24th AIAA Fluid Dynamics Conference, Orlando, Florida (July 1993).

[41] N. Melson, D. Sanetrik, H. Atkins, Time-accurate Navier-Stokes Calculations with Multigrid Acceleration, Proc. $6^{\text {th }}$ Copper Mountain Conference on Multigrid Methods (1993) II423-II437. 
[42] M. Campobasso, M. Baba-Ahmadi, Ad-hoc Boundary Conditions for CFD Analyses of Turbomachinery Problems with Strong Flow Gradients at Farfield Boundaries, Journal of Turbomachinery 133 (4).

[43] M. Raciti-Castelli, A. Englaro, E. Benini, The Darrieus wind turbine: proposal for a new performance prediction model based on cfd, Energy 36 (2011) 4919-4934.

[44] M. Raciti-Castelli, G. Pavesi, L. Battisti, E. Benini, G. Ardizzon, Modeling strategy and numerical validation for a Darrieus vertical axis microwind turbine, ASME paper IMECE-2010-39548, aSME 2010 International Mechanical Engineering Congress and Exposition, Vancouver, Canada (November 2010).

[45] F. Balduzzi, A. Bianchini, F. Gigante, G. Ferrara, M. Campobasso, L. Ferrari, Parametric and Comparative Assessment of Navier-Stokes CFD Methodologies for Darrieus Wind Turbine Performance Analysis, ASME paper GT2015-42663, aSME Turbo Expo 2015, Gas turbine Technical Conference and Exposition, Montreal, Canada (June 2015).

[46] Ansys, Fluent theory guide, Tech. Rep. release 14.5 (2013).

[47] M. Campobasso, M. Yan, J. Drofelnik, A. Piskopakis, M. Caboni, Compressible Reynolds-Avergaed Navier-Stokes Analysis of wind Turbine Turbulent Flows Using a Fully Coupled Low-Speed Preconditioned Multigrid Solver, ASME paper GT2014-25562 (June 2014).

[48] M. Campobasso, M. Giles, Effects of flow instabilities on the linear analysis of turbomachinery aeroelasticity, Journal of Propulsion and Power 19 (2) (2003) 250-259.

[49] M. S. Campobasso, M. B. Giles, Stabilization of Linear Flow Solver for Turbomachinery Aeroelasticity Using Recursive Projection Method, AIAA Journal 42 (9) (2004) 1765-1774. 
${ }_{1210}$ [50] K. Ekici, K. Hall, H. Huang, J. Thomas, Stabilization of Explicit Flow Solvers Using a Proper-Orthogonal-Decomposition Technique, AIAA Journal 51 (5) (2013) 1095-1104.

[51] Z. Yang, D. Mavriplis, Time Spectral Method for Periodic and QuasiPeriodic Unsteady Computations on Unstructured Meshes, AIAA paper 2010-5034, 40th Fluid Dynamics Conference and Exhibit, Chicago, Illinois (June-July 2010). 